\title{
A Review of Graphene Plasmons and its Combination with Metasurface
}

\author{
Chuanbao Liu*, Yang Bai**, Ji Zhou**, Qian Zhao***;, and Lijie Qiao* \\ *Key Laboratory of Environmental Fracture (Ministry of Education), University of Science and Technology Beijing, \\ Beijing 100083, China \\ ${ }^{* *}$ State Key Laboratory of New Ceramics and Fine Processing, Tsinghua University, Beijing 100084, China \\ *** State Kay Laboratory of Tribology, Department of Mechanical Engineering, Tsinghua University, Beijing 100084, China
}

(Received July 23, 2017; Revised September 5, 2017; Accepted September 6, 2017)

\begin{abstract}
Graphene has attracted a lot of attentions due to the unique electrical and optical properties. Compared with the noble metal plasmons in the visible and near-infrared frequencies, graphene can support surface plasmons in the lower frequencies of terahertz and mid-infrared and it demonstrates an extremely large confinement at the surface because of the particular electronic band structures. Especially, the surface conductivity of graphene can be tuned by either chemical doping or electrostatic gating. These features make graphene a promising candidate for plasmonics, biosensing and transformation optics. Furthermore, the combination of graphene and metasurfaces presents a powerful tunability for exotic electromagnetic properties, where the metasurfaces with the highly-localized fields offer a platform to enhance the interaction between the incident light and graphene and facilitate a deep modulation. In this paper, we provide an overview of the key properties of graphene, such as the surface conductivity, the propagating surface plasmon polaritons, and the localized surface plasmons, and the hybrid graphene/metasurfaces, either metallic and dielectric metasurfaces, from terahertz to near-infrared frequencies. Finally, there is a discussion for the current challenges and future goals.
\end{abstract}

Key words : Electrical properties, Optical properties, Sensors

\section{Introduction}

$\mathrm{G}$ raphene, a single layer of carbon atoms arranged in Thoneycomb structures, has attracted extensive attentions since the advent of free-standing graphene sheets in 2004. ${ }^{1)}$ Because of the linear dispersion relation of Dirac electronic states, graphene demonstrates the unique electrical and optical properties, ${ }^{2,3)}$ such as high carrier mobility, broadband operation, and a tunable band gap, which make it a fantastic material for photonics and optoelectronics. ${ }^{4-6)}$ The optical response of graphene can be described by the surface conductivity which is originated from the contributions of inter-band and intra-band transitions, related to the chemical potential or Fermi level. ${ }^{7,8)}$ In the near-infrared and visible frequencies, the graphene manifests a strong absorption caused by the inter-band transitions. ${ }^{7,9)}$ In the terahertz and mid-infrared frequencies, the graphene behaves as an ultrathin metal film, supporting the surface plasmon polaritons (SPPs) with the greatly enhanced local fields at the graphene surface. ${ }^{10-13)}$ Compared with the conventional metal palsmons primarily working in the visible frequencies, the SPPs in graphene demonstrate a stronger confinement and lower loss, that is, an extremely short wavelength and a long propagation distance. ${ }^{12)}$ Most import-

\footnotetext{
Corresponding author: Yang Bai

E-mail : baiy@mater.ustb.edu.cn

Tel : +86-10-6233-4493 Fax : +86-10-6233-4493
}

ant, SPPs in graphene can be dynamically tuned by the chemical doping ${ }^{14)}$ or electrostatic gating. ${ }^{15)}$ As a result, graphene has promising applications for high performance SPP-based nano-devices. ${ }^{16)}$

Due to the large mismatch between the wave vector of SPPs and the incident light, many methods, such as nanoemitters/tips scattering, ${ }^{12,13,17-23)}$ grating ${ }^{24-27)}$ and prism coupling, ${ }^{28,29)}$ have been proposed. However, the coupling efficiency in the above methods is very low. Alternatively, the structured graphene (sub-wavelength nanoparticles) can demonstrate localized surface plasmons (LSPs) resonance with a strongly confined local field at the surface of nanoparticles. ${ }^{30-37)}$ Compared with the propagating SPPs, LSPs resonance is a non-propagating regime and can be directly excited by a light with appropriate polarization and frequency. Apart from the strong light-matter interaction for sensing applications, the structured graphene is capable of modulating phase and amplitude used in polarization converter, ${ }^{38,39)}$ optical activity ${ }^{40)}$ and wavefront reshaping applications. ${ }^{41-44)}$

The optical response for the monolayer graphene usually has a limited modulation depth due to the fact of single atomic thickness. To obtain a more powerful capacity of modulation, the hybrid graphene/metasurfaces have been proposed as a possible solution. ${ }^{45-48)}$ Metasurfaces are composed of sub-wavelength scatters which can interact with the electric or magnetic field components of incident light to produce the desired responses, including the phase shift, 
amplitude and polarization. By arranging the spatial distribution of the designed scatters treated as the tiny secondary sources, the metasurface can arbitrarily manipulate the electromagnetic wavefront, leading to myriad applications, such as holograms, ${ }^{49-51)}$ meta-lens ${ }^{52-55)}$ and abnormal refraction. ${ }^{56-58)}$ Compared with the conventional optical components or bulky metamaterials ${ }^{59,60)}$ depending on the electromagnetic wave propagating in the bulky media to gradually accumulate the changes of wavefront, the metasurfaces can realize the abrupt changes in a sub-wavelength-thin thickness, endowing the superior advantages of miniaturization, lightening and thinning. A drawback of conventional metasurfaces is non-tunability after they were fabricated. The versatile functionality of metasurfaces depends on the unit cells which are implemented by metallic patterns or dielectric cubes based on the plasmonic or Mie resonances. ${ }^{56,61-63)}$ It opens up an avenue to dynamically control light when the active graphene integrates with metasurfaces, which demonstrates more extensive application prospects, such as fast optical modulators, ${ }^{46,64-67)}$ switches ${ }^{45,47)}$ and other optical devices. ${ }^{68,69)}$ In addition, graphene is compatible with the nanofabrication technology and easy to be integrated into the nano/micro-sized metasurfaces.

This paper is organized as described below. In section 2 , we overview the surface conductivity of graphene from terahertz to visible frequencies by the random-phase approximation (RPA) theory. In section 3, we introduce the propagating SPPs in graphene, including the characteristics, SPPs excitation, and SPPs hybridization. In section 4, we introduce LSPs in the structured graphene, modulation depth of absorption and electromagnetic applications, such as polarizer and meta-lens. In section 5 , we overview the hybrid graphene/metallic metasurface working from terahertz to near-infrared frequencies, and the hybrid graphene/ dielectric metasurface mainly working in the near-infrared frequencies. In the last section, we provide some concluding remarks and present an outlook on future research directions.

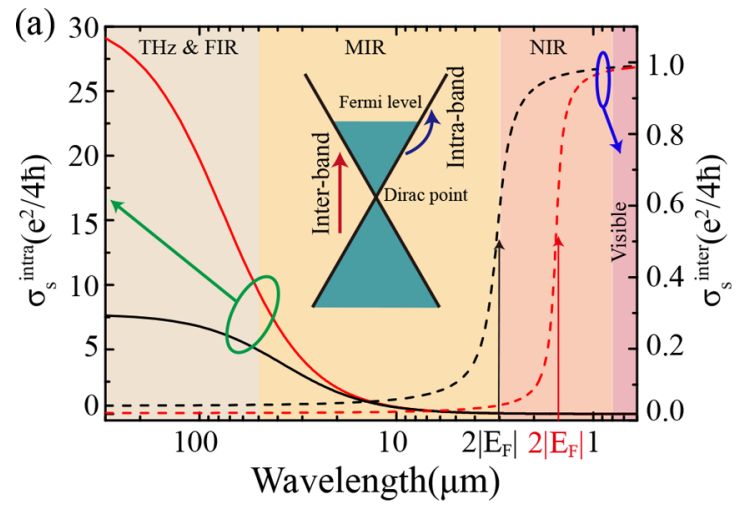

\section{Surface Conductivity of Graphene}

\subsection{Surface conductivity at terahertz and infrared frequencies}

The optical response of graphene is derived from the dynamic surface conductivity closely related to chemical potential or Fermi level. It can be derived with the randomphase approximation (RPA) theory ${ }^{8)}$ and originate from the contribution of intra-band and inter-band transitions as below,

$$
\begin{aligned}
& \sigma_{s}(\omega)=\frac{i \cdot 2 e^{2} k_{B} T}{\pi \hbar^{2}\left(\omega+i \tau^{-1}\right)} \ln \left[2 \cosh \left(\frac{E_{F}}{2 k_{B} T}\right)\right] \\
& +\frac{e^{2}}{4 \hbar}\left[\frac{1}{2}+\frac{1}{\pi}+\arctan \left(\frac{\hbar \omega-E_{F}}{2 k_{B} T}\right)-\frac{i}{2 \pi} \ln \frac{\left(\hbar \omega+E_{F}\right)^{2}}{\left(\hbar \omega-E_{F}\right)^{2}+\left(2 k_{B} T\right)^{2}}\right]
\end{aligned}
$$

where $e$ is the electron charge, $k_{B}$ is the Boltzmann's constant, $\hbar$ is the reduced Planck's constant, $T$ is the temperature, and $\omega$ is the angular frequency of incident light. $\tau$ is the relaxation time and estimated by expression $\tau=u E_{F} / e v_{f}^{2}$, where $v_{f}=10^{6} \mathrm{~m} / \mathrm{s}$ is the Fermi velocity, $u$ is the carrier mobility of graphene and ranges from $1000 \mathrm{~cm}^{2} / \mathrm{Vs}$ in chemical vapor deposition $(\mathrm{CVD})^{70,71)}$ to $100 \mathrm{~m}^{2} / \mathrm{Vs}$ in suspended graphene. ${ }^{72)} E_{F}$ is the Fermi level which translates to the carrier concentration of $n=E_{F}^{2} / \pi \hbar^{2} v_{f}^{2}$. In most cases, the Fermi energy $E_{\mathrm{F}}$ is limited range of $-1.0 \mathrm{eV}$ to $1.0 \mathrm{eV}$ as the consideration of device requirements to guarantee against the breakdown electric field of the gate dielectric. ${ }^{73)}$ The first and second terms are attributed to the intra- and inter-band transitions, respectively.

Figure 1 is the calculated results based on Eq. (1). In the terahertz and mid-infrared frequencies where the photonic energy is far below the double Fermi level ( $\hbar \omega<<2\left|E_{F}\right|$ ), the surface conductivity of graphene are dominated by the intra-band transitions (Fig. 1(a)). The inter-band transitions are diminished due to the presence of electrons and holes near the band edges (Pauli blocking). The surface conductivity can be reduced to

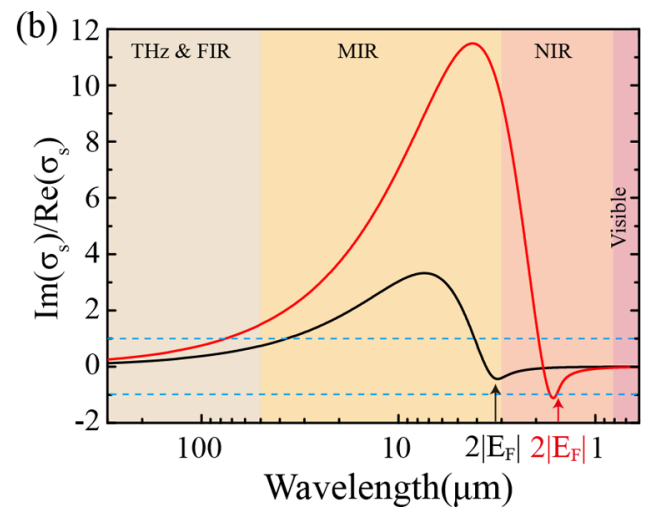

Fig. 1. (a) Real parts of intra-band (solid) and inter-band (dash) surface conductivities of graphene with $E_{F}=0.2 \mathrm{eV}$ (black) and $E_{F}=0.4 \mathrm{eV}$ (red). The carrier mobility is assumed to be $\mu=1000 \mathrm{~cm}^{2} / \mathrm{Vs}$. The inset shows the band structure of graphene. (b) The optical response of moderately doped graphene in different spectral ranges. In the terahertz, near-infrared or midinfrared frequencies, graphene exhibits a lossy $\left(\left|\operatorname{Im}\left(\sigma_{s}\right) / \operatorname{Re}\left(\sigma_{s}\right)<1\right|\right)$ or plasmonic behavior $\left(\operatorname{Im}\left(\sigma_{s}\right) / \operatorname{Re}\left(\sigma_{s}\right)>1\right)$, respectively. 


$$
\sigma_{s}(\omega)=\frac{i \cdot 2 e^{2} k_{B} T}{\pi \hbar^{2}\left(\omega+i \tau^{-1}\right)} \ln \left[2 \cosh \left(\frac{E_{F}}{2 k_{B} T}\right)\right] .
$$

When the Fermi level is $E_{F}>k_{B} T$, the surface conductivity is further simplified to

$$
\sigma_{s}(\omega)=\frac{i \cdot e^{2} E_{F}}{\pi \hbar^{2}\left(\omega+i \tau^{-1}\right)} .
$$

From Eqs. (2) and (3), the graphene just behaves as a conductive film and can be described by a classical Drude model. As a result, the graphene can support the transverse magnetic (TM) polarized SPPs. The surface conductivity presents a linear relationship with the Fermi level $E_{F}$. Note that a considerable loss caused by the intra-band transitions is inevitable in the terahertz frequencies (Fig. 1(b)) which can be utilized to modulate amplitude by the absorption in graphene instead.

However, in the mid-infrared frequencies, the surface conductivity approaches the minimum due to the Pauli blocking and a relatively large incident frequency, degenerating the inter-band and intra-band transitions (Fig. 1(a)). The imaginary part of conductivity becomes dominant over the real part, facilitating to support the transverse magnetic SPPs with an extremely low loss (Fig. 1(b)). In the terahertz and mid-infrared frequencies, the conductivity of graphene has a largely tunable range which is facilitated to largescalely tune the amplitude or frequency of the plasmon resonance when the graphene critically couples with the metasurface of enhanced local field. Besides, the very thin "metal" layer can be further tailored to different shapes to obtain the desired resonances and reshape wavefront.

For the numerical simulations, graphene is often modeled as an anisotropic medium with in-plane effective permittivity as

$$
\varepsilon_{e f f, t}=1+\frac{i \sigma_{s}}{\varepsilon_{0} \omega t_{g}}=1-\frac{\omega_{p}^{2}}{\omega^{2}}
$$

where $\varepsilon_{0}$ is the vacuum permittivity, $t_{g}$ is the thickness of graphene, $\omega_{p}=\left(\frac{2 e^{2} k_{B} T}{\pi \hbar^{2} \varepsilon_{0} t_{g}} \ln \left[2 \cosh \left(\frac{E_{F}}{2 k_{B} T}\right)\right]\right)^{1 / 2}$ is the plasm frequency. The out-plane effective permittivity is equal to the vacuum permittivity of $\varepsilon_{\text {eff }, n}=1.0$.

\subsection{Surface conductivity at near-infrared and visi- ble frequencies}

On the contrary, the inter-band transitions become a decisive factor $\left(\hbar \omega>2\left|E_{F}\right|\right)$ in the near-infrared and frequencies, as shown in Fig. 1(a). The free carrier scattering rate $\tau^{-1}$ can be treated as zero due to the small quantity compared with the incident frequency. ${ }^{74)}$ The real part of the surface conductivity is changed from 0 to $e^{2} / 4 \hbar$ (universal optical conductivity) by electrically tuning Fermi level, which results in a modulation of absorption from 0 to $2.3 \%$ under the normal incidence. Obviously, the limited range of surface conductivity also limits the modulation depth of an isolated graphene, no matter for the transmission, reflection and absorption. ${ }^{75)}$ To get a large modulation, the metasurface which can capture light from free space and offer a local filed enhancement in plane (two to three orders of magnitude larger than the incident field) is critical coupling with the tunable graphene. Besides, the imaginary part of the graphene permittivity becomes dominant over the real part so that the surface plasmons cannot be sustained at the graphene surface. ${ }^{10)}$ In the visible frequencies, graphene can be visualized despite of only a single atom thick, due to a significant absorption $\pi \alpha=2.3 \%$, where $\alpha=1 / 137$ is the fine structure constant that describes coupling between light and electrons.

It is noted that the surface conductivity can also be tuned by the photonic excitation, ${ }^{76)}$ magnetic field ${ }^{77)}$ and strain mechanism, ${ }^{78)}$ in addition to the common electrostatic gating and chemical doping.

\section{Propagating Surface Plasmon Polaritons in Graphene}

\subsection{Properties of SPPs}

The SPP is a collective oscillation of conduction electrons or holes along the boundary surface of a metal or semiconductor under the drive of electric field component of an incident light. It can highly confine the plasmonic wave and strongly enhance the electric field in the vicinity of surface, promising applications for the light-mater interactions, such as chemical, biological sensing and surface-enhanced Raman scattering. ${ }^{79)}$ As stated above, the graphene behaves as a metal film in the terahertz and infrared frequencies, i.e. it is capable of supporting transverse magnetic polarized SPPs. For the simplest dielectric/continuous graphene sheet (the size larger than SPPs wavelength)/dielectric prototype, the dispersion relationship of SPPs is given by ${ }^{10,80)}$

$$
\beta(\omega)=\frac{i\left(\varepsilon_{r 1}+\varepsilon_{r 2}\right) \varepsilon_{0} \omega}{\sigma_{s}(\omega)}=\frac{\pi \hbar^{2} \varepsilon_{0}\left(\varepsilon_{r 1}+\varepsilon_{r 2}\right)}{e^{2} E_{F}} \omega\left(\omega+i \tau^{-1}\right),
$$

where $\beta$ is the wave-vector of SPPs in graphene, $\varepsilon_{r 1}$ and $\varepsilon_{r 2}$ are the permittivity of dielectric above and below the graphene. Note that the precondition for the establishment of Eq. (5) is $k_{0}<\beta<<k_{F}$ (in non-retarded regime and long wavelength limit), where $k_{0}$ and $k_{F}$ are the vacuum and Fermi wave-vectors. For a finite $\beta$ compared with $k_{F}$, the dispersion of SPPs will deviate the Eq. (5) and present a linear relationship or damping regime. ${ }^{81)}$ In addition, the dispersion of SPPs is also influenced by the polar medium, where the surface optical phonons couple with the SPPs via a long-range Fröhlich coupling. ${ }^{82)}$

Compared with the conventional noble metal (silver and gold) plasmons, the graphene supported SPPs have many prominent properties. (1) Huge modal index or extensive confinement. For clarity, the Eq. (5) is simplified to

$$
\beta(\omega) \approx \frac{\left(\varepsilon_{r 1}+\varepsilon_{r 2}\right) \hbar \omega}{4 \alpha E_{F}} k_{0},
$$


where the scattering rate $\tau^{-1}$ is assumed to zero, $\alpha=e^{2}$ / $4 \pi \hbar \varepsilon_{0} c=1 / 137$ is the fine structure constant, the weight of $\hbar \omega / E_{F}$ is order of 1 . Hence, the wave-vector of SPPs in graphene is two orders of magnitude bigger than the vacuum wave-vector. ${ }^{13,19,20)}$ The wave-vector of SPPs in the noble metal, by contrast, is slightly larger than the vacuum wave-vector. $^{83)}$ (2) Relatively low losses or longer propagation. In the mid-infrared frequencies, the surface conductivity reaches the minimum, as shown in Fig. 1. The real part of permittivity in the graphene dominate the imaginary part, resulting in a relatively low damping loss of SPPs and a longer propagation length about hundreds of wavelength of SPPs, comparing to a range of tens of optical cycle for SPPs on metals. (3) Dynamic tunability. From Eq. (5), the propagation of SPPs in graphene is related to the Fermi level which can be tuned by electric field, magnetic field, electrostatic gating and chemical doping in real time. (4) Broadband operation. For the SPPs at the metal/dielectric surface which only works for the frequencies close to the plasma frequency of metal. At terahertz and infrared frequencies, the metal behaves as a perfect conductor with delocalized SPPs. On the contrary, the SPPs can be supported by the graphene from terahertz to near-infrared frequencies by controlling the Fermi level. These unique features make the graphene a promising platform for the active SPP-based nano-optoelectronic devices, plasmonics and transformation optical devices by spatially varying the graphene Fermi level in the terahertz and infrared frequencies. $^{84)}$

\subsection{SPPs excitation}

The major challenge is to efficiently excite SPPs in graphene with a free space incident light due to the large wave-vector mismatch between two waves. Conventional plasmonics approaches, such as the diffractive grating, ${ }^{24-27)}$ shifts the wave-vector of the incident light to compensate the mismatch due to Bragg scattering following the expression of $\beta=\sin \theta k_{0}+2 n \pi / \Lambda$, where $n$ is the diffraction order, $\Lambda$ is the periodicity, $\theta$ is the incident angle. Fig. 2(a) and (b) show the silicon-diffractive grating-assisted graphene plasmon excitation by guided-wave resonance. ${ }^{24)}$ The Fourier transform infrared spectroscopy (FTIR) measurement result of $T_{+} / T_{/ /}$, where $T_{+}$and $T_{/ /}$are the transmission spectrum under the normal incidence polarized perpendicular and parallel to the gratings lines, demonstrates a resonant dip due to the absorption of SPPs in the graphene (Fig. 2(c)). It is noted that, to efficiently excite the SPPs, a large coupling length is required (in the order of incident wavelength), which goes against the compactness of the nanodevices and bring a challenge to the nanofabrication.

Another conventional method to excite the SPPs is using the prism coupling, ${ }^{28,29)}$ such as the Otto or Kretschmann configurations, ${ }^{85,86)}$ where the evanescent wave propagates along the metal-dielectric interface, due to the total internal reflection under the condition of the incident angle $(\theta)$ larger than the critical angle $\left(\theta_{c}\right)$. However, the huge difference of wave-vector between the evanescent wave and the incident light requires a high refractive index of the prism $\left(n_{p} \approx 10-100\right)$, according to the equation $\beta=k_{0} n_{p} \sin \theta$, which is not available practically. Nikitin et al. ${ }^{29)}$ proposed a tactful configuration (Fig. 2(d)) to excite the SPPs in graphene based on the compression of surface polaritons (SPs) on the tapered slabs of a polaritonic material supporting either surface phonon polaritons (SPhPs) or SPPs, as shown in Fig. 2(e) and (f). The SPhPs or SPPs generating by the prism coupler propagate along the tapered slab and gradu- (a)

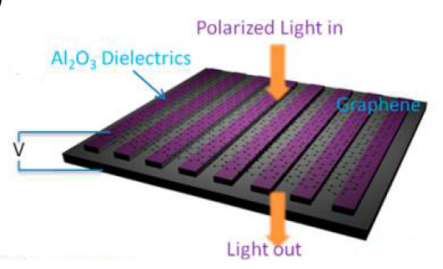

(b)

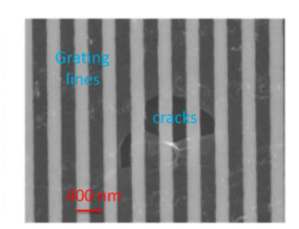

(c)

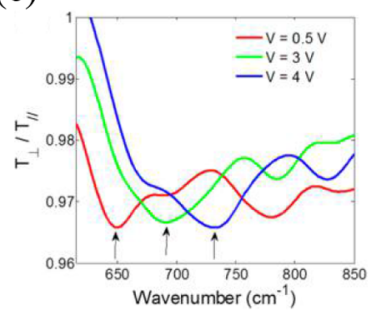

(f)

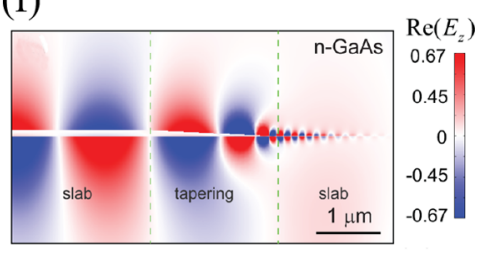

Fig. 2. (a) Schematic of silicon-diffractive grating-assisted graphene plasmon excitation by guided-wave resonance (GWR). (b) Scanning electron microscopy (SEM) image of transferred graphene on a silicon grating, where the dark regions are the cracks. (c) Electrical tuning of the fabricated GWR graphene plasmonic device where arrows indicate the position of the resonant dips. (d) Schematic of the proposed coupling device, consisting of an Otto prism (couple the incident light to SPs), a tapered slab (compress the wavelength of SPs) and a thin slab (couple the SPs to SPPs in graphene). (e, f) Snapshots of the real part of the vertical component of the electric field $\operatorname{Re}\left(\mathrm{E}_{\mathrm{z}}\right)$ for $\mathrm{SiC}$ and $\mathrm{n}$-GaAs waveguides. (a) - (f) used with permission from ${ }^{24,29)}$ copyright by 2013, 2014 by American Chemical Society. 
(a)

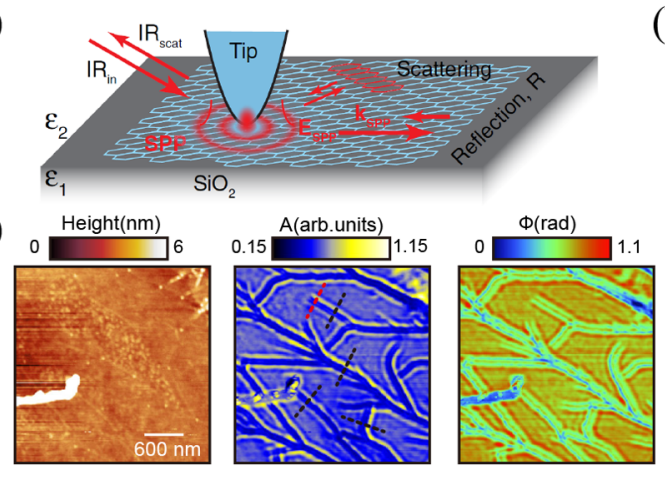

(b)

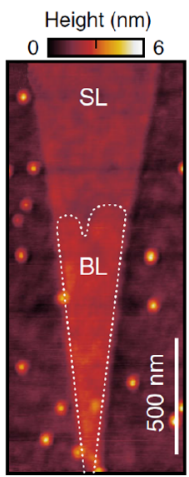

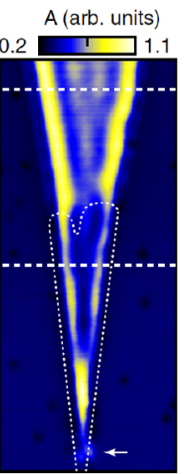

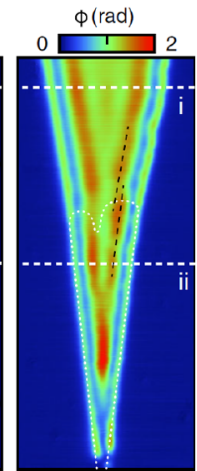

(d)

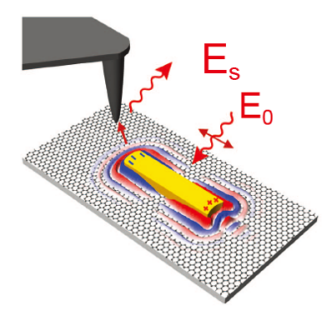

(e)

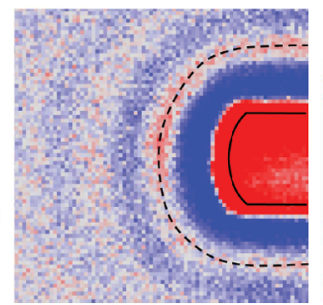

(f)

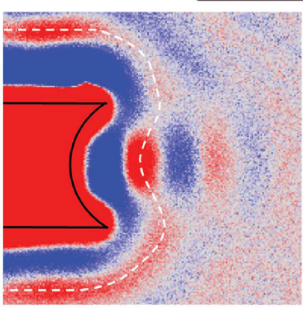

$\operatorname{Re}\left(E_{s, p}\right)$

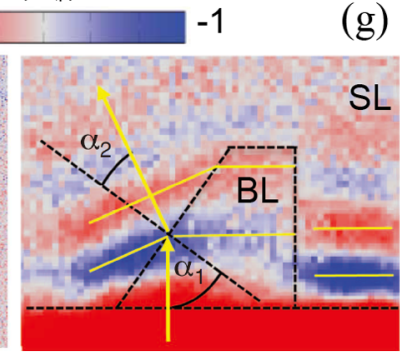

Fig. 3. (a) Illustration of metallic tip-induced SPP excitation and subsequent interference due to the emission of scattered and reflected SPP waves. (b, c) AFM topography (left), near-field amplitude (middle), and near-field phase (right) of a graphene wedge and a region of single-layer graphene with a high concentration of grain boundaries and folds. (d) Schematic of an antenna-based graphene SPPs launcher. (e, f) Experimental near-field images $\operatorname{Re}\left(\mathrm{E}_{\mathrm{s}, \mathrm{p}}\right)$ of a convex and a concave antenna extremity, respectively $\left(\lambda_{0}=11.06 \mu \mathrm{m}\right)$. $(\mathrm{g})$ Experimental near-field image $\operatorname{Re}\left(\mathrm{E}_{\mathrm{s}, \mathrm{p}}\right)$ of a bilayer graphene prism on a monolayer graphene $\left(\lambda_{0}=10.20 \mu \mathrm{m}\right)$. (a) - (c) used with permission from ${ }^{20)}$ copyright 2014 by American Physical Society, (d) - (g) used with permission from ${ }^{87)}$ copyright 2014 by AAAS.

ally yield a compression of wavelength to match the SPPs in graphene finally.

Besides the above methods, the excitation of SPPs is also proposed by coupling the near field of the nano-emitters with graphene. ${ }^{12,13,17-23)}$ Typically, the metallized tip of an atomic force microscope (AFM) illuminated by a focused infrared laser beam generates strong evanescent electric fields with a wide range of in-plane momenta which can effectively excite the graphene plasmons. In combination with the scattering-type scanning near-field optical microscopy (s-SNOM), ${ }^{13,19,20,23)}$ the SPPs reflected or scattered by the external and internal edges, atomic defects, and boundaries of single-layer or multilayer graphene are visualized including amplitude and phase. Fig. 3(a) shows the illustration of the tip-induced SPPs excitation. ${ }^{20)}$ Under the illumination of a $\mathrm{CO}_{2}$ laser with a wavelength of $\lambda=10.8 \mu \mathrm{m}$, the s-SNOM amplitude and phase exhibits the standing waves for SPPs due to the existence of external (transition from single and bilayer graphene) and internal (folds and grain boundaries) defects. The SPPs wavelength $\left(\lambda_{s p p}\right)$ in single and bilayer graphene are $(260 \pm 10) \mathrm{nm}$ and $(190 \pm 10) \mathrm{nm}$, with a reflection coefficient of $\tilde{R}=-1$, respectively (Fig. 3(b)). The SPPs in both sides of the line defects have different wavelength of $240 \mathrm{~nm}$ and $260 \mathrm{~nm}$, as well as different reflection coefficients of $\tilde{R}=0.45$ and 0.55 (Fig. 3(c)). The ratio of $\lambda_{s p p} / \lambda_{0}$ is in the order of the fine structure constant. Besides, the change of Fermi level can effectively tune the SPPs wavelength. The full amplitude and phase spatially resolved imaging provides nano-scale insight into the local electronic structure and dynamics in graphene.

Figure 3(d) demonstrates the propagating SPPs in graphene excited by the dipole nano-antenna under the incident light $\left(\lambda_{0}=10.20 \mu \mathrm{m}\right.$ or $\left.11.06 \mu \mathrm{m}\right)$ polarized parallel to the long axis and imaged by s-SNOM with a dielectric Si-tip which passively scatter the near field of the graphene plasmon and gather the cross-polarization with a polarizer in front of the detector. ${ }^{87)}$ The study shows the efficiency of resonance is enhanced by a factor of 28 compared with the nonresonant antennas. Besides, the wavefront of SPPs can be engineered by the shape of resonant antennas, such as concave and convex extremities (Fig. 3(e) and (f)). Further, the author demonstrated the propagating direction of SPPs can be controlled by a $2 \mathrm{D}$ graphene bilayer prism based on the conductivity patterns ${ }^{84)}$ and revealed that the wavenumber in graphene is also influenced by the number of graphene layers $\left(\lambda_{s p p} \sim N^{-1 / 2}, N\right.$ is the number of layers) which qualitatively followed the Snell' law (Fig. 3(g)). ${ }^{37}$

Furthermore, the SPPs in graphene can be visualized by the electron energy-loss (EEL) spectrum imaging ${ }^{22}$ at the atomic scale which offers a platform to study the effect of 
(a)

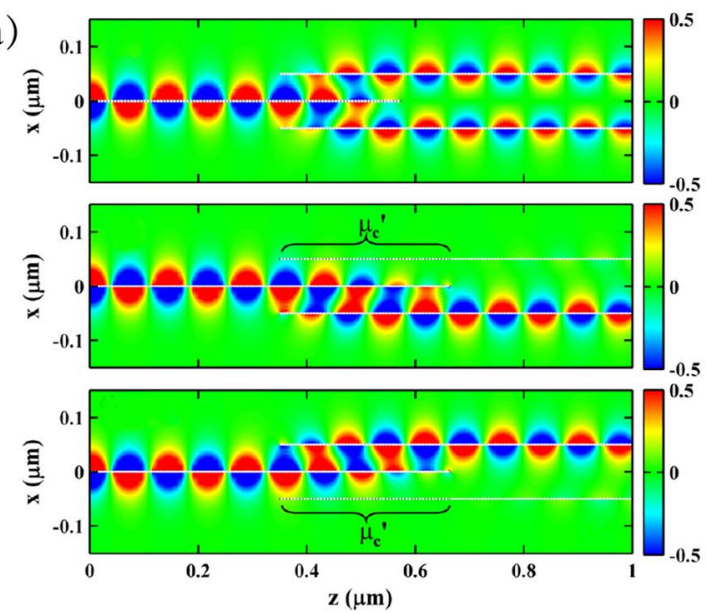

(b)

(c)
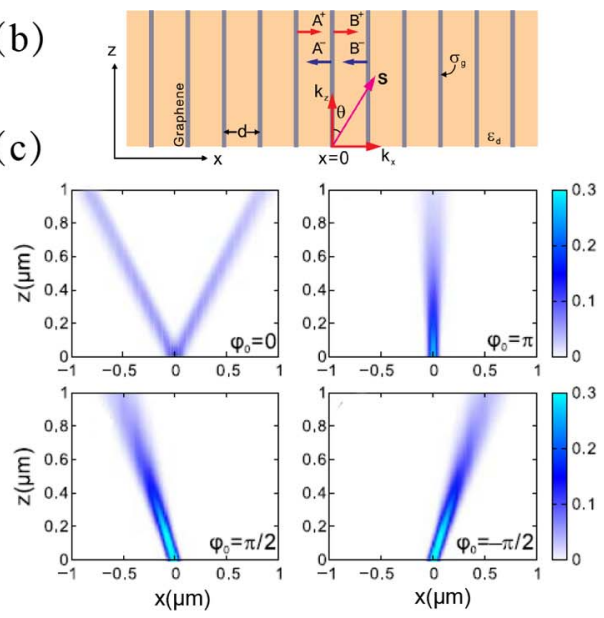

Fig. 4. (a) Electric field ( $E_{x}$, perpendicular to graphene surface) distribution of SPPs with bias on the output arm by a gate voltage: (top) no bias as splitter, upper arm (middle) or lower arm (bottom) biased for digital switch. (b) Schematic of a monolayer graphene sheet array (MGSA). (c) Intensity distributions of SPPs in the MGSA for different initial phase differences $\left(\varphi_{0}\right)$ of SPPs between neighboring graphene. (a) used with permission from ${ }^{88)}$ copyright 2012 by AIP Publishing, (b) and (c) used with permission from ${ }^{89)}$ copyright 2012 by American Physical Society.

single atom on monolayer graphene sheets and brings the possibilities for designing atomic-scale optoelectronic and plasmonic devices.

\subsection{SPPs hybridization}

So far, we mainly review the excitation of SPPs in monolayer graphene sheet with a large size compared with the plasmon wavelength. Actually, the interaction of spatially separated graphene sheets is also an important subfield of graphene plasmon, because it is not only a fundamental issue but also provides a wider range of application prospects. Fig. 4(a) shows a graphene waveguide splitter. ${ }^{88)}$ The propagating SPPs in the high doped graphene $\left(\hbar \omega<<E_{F}\right)$ are completely transferred to the adjacent graphene by the weak SPPs coupling in the coupling region (top, Fig. 4(a)). By decreasing the Fermi level of the graphene in the upper or low coupling region to the condition of $2\left|E_{F}\right|<\hbar \omega$, where the graphene no longer supports SPPs (middle and bottom, Fig. 4(a)), the configuration realizes a tunable optical splitter or switch. Then, the author studied the strong coupling of SPPs in the monolayer graphene sheet arrays (MGSA) (Fig. 4(b)), ${ }^{89)}$ which are physically different from the above case with weak coupling and demonstrate interesting properties (Fig. 4(c)). Beyond that, the SPPs in the monolayer graphene ribbon and the plasmonic hybridization of the spatially separated graphene ribbons are also intensively studied. ${ }^{90,91)}$ The graphene ribbon supports an edge SPPs (ESPPs) with a strongly localized field at the ribbon edges except the waveguide SPPs with the field concentration within the ribbon area. Especially, the graphene ribbon pairs demonstrate a more intensive enhancement of field. ${ }^{91)}$

Benefiting from the fascinating properties of SPPs in graphene in the terahertz and mid-infrared frequencies, the graphene opens up an exciting opportunity for the active sub-wavelength optics and the extremely compact optoelec- tronic devices.

\section{Localized Surface Plasmons in Structured Graphene}

\subsection{Properties of LSPS}

Different from the propagating SPPs, the LSPs are the non-propagating excitation of conductions of sub-wavelength metallic particles coupled with the incident light. It can be resonantly excited with a light of appropriate frequency and polarization, irrespective of the wave-vector match required in the propagating SPPs. At the plasmon resonant frequency, the LSPs are strongly confined to the surface of sub-wavelength particles and result in a significant electric field enhancement, facilitating to capture the incident light in solar cell and light-mater interaction for sensing applications. In the terahertz and mid-infrared frequencies, the noble metals are too metallic so that the metallic micro-structures suffer from a relatively poor field confinement, going against the non-destructive sensing. Alternatively, the graphene, as an excellent plasmonic material with an adjustable plasma frequency, can be structured into different shapes such as micro/nano-ribbons, ${ }^{30-34)}$ nano-tubes $^{35)}$ and disks ${ }^{36,37)}$ which are capable of exploring the tunable LSPs in the terahertz and infrared frequencies.

Figure 5(a) and (b) are the graphene micro-ribbons ${ }^{32)}$ and nano-ribbons ${ }^{34)}$ tuned by the electrostatic electrolyte-gating and back-gating configurations working in the terahertz and mid-infrared frequencies, respectively. The extinction spectral in transmission is defined as $1-T / T_{R}$, where $T$ is the transmission through the graphene ribbons with an electric field perpendicular to the ribbons, $T_{R}$ is the referenced transmission with different types, such as transmission through substrate without graphene ribbons, ${ }^{34)}$ transmission through graphene ribbons at the charge neutral point 
(a)

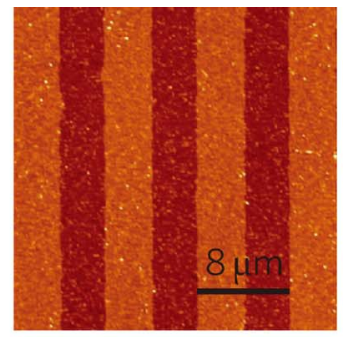

(c)

(g)
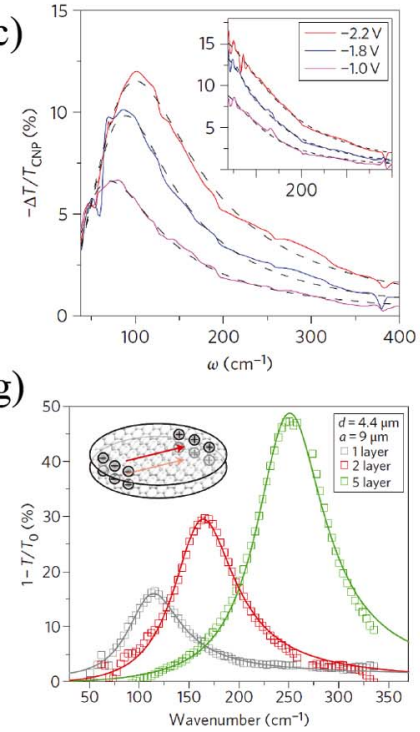

(b)
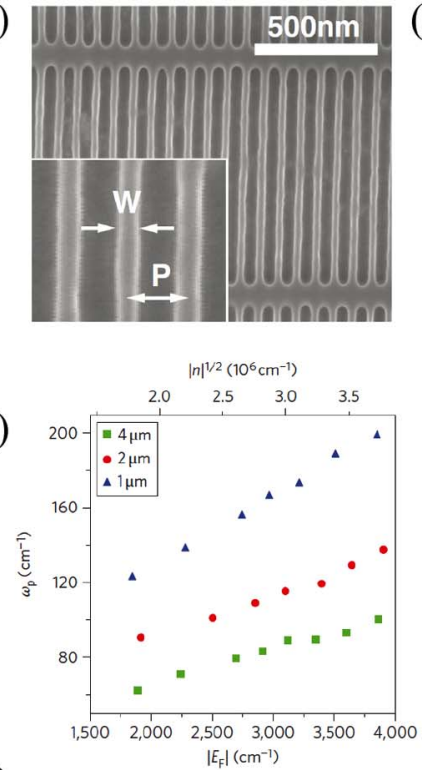

(h)

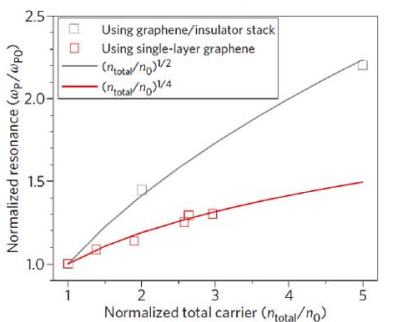

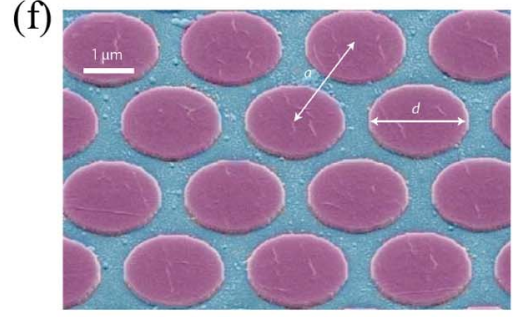

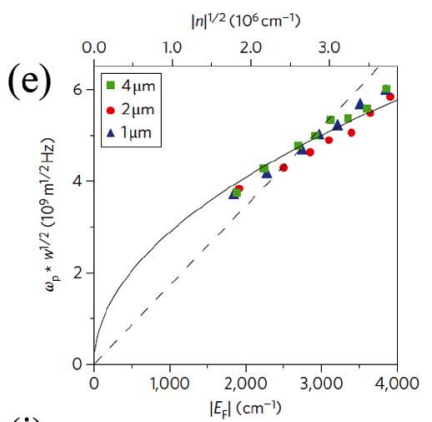

(i)

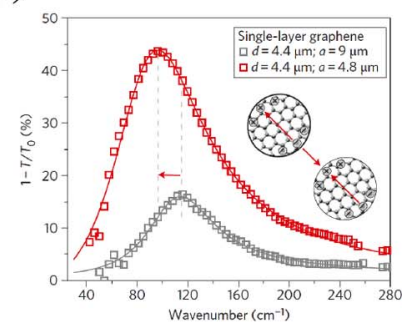

Fig. 5. (a) AFM and (b) SEM images of a graphene micro-ribbon array and a nano-ribbon array. (c) Control of terahertz resonance of plasmon excitations through electrical gating. Terahertz radiation was polarized perpendicular to the graphene ribbons. The inset shows extinction spectra for terahertz radiation polarized parallel to the ribbons. (d) Plasmon resonance frequency $\omega_{p}$ as a function of $\left|E_{F}\right|$ or $|n|^{1 / 2}$ for micro-ribbon arrays of different widths. (e) Normalized plasmon resonance frequency $\omega_{p} w^{1 / 2}$ as a function of $\left|E_{F}\right|$ or $|n|^{1 / 2}$ for micro-ribbon arrays of different widths. (f) SEM image (false color) of a stacked graphene/insulator micro-disk array arranged in a triangular lattice. (g) Extinction spectra for stacked plasmonic devices with one, two and five graphene layers under normal incidence. (h) Normalized plasmonic resonance frequency versus normalized total carrier density for single-layer graphene and graphene/insulator graphene stack. (i) Extinction spectra in single-layer graphene plasmonic devices with identical $d(4.4 \mu \mathrm{m})$ but different $a(4.8$ and $9 \mu \mathrm{m}$, respectively). Inset: schematic of the graphene disk-disk interaction. (a) and (c) - (e) used with permission from ${ }^{32)}$ copy- $^{-}$ right 2011 by Nature Publishing, (b) used with permission from ${ }^{34)}$ copyright 2015 by AAAS, (f) - (i) used with permission from $^{37)}$ copyright 2012 by Nature Publishing.

$(\mathrm{CNP})^{32)}$ and transmission through graphene ribbons with electric fields parallel to the ribbons. ${ }^{33)}$ The extinction spectral indicates a strong absorption and exhibits a prominent peak at the LSPs resonance. The frequency and strength of the maximum absorption can be tuned by the bias voltage, which clearly demonstrates a plasmon tunability through electrical gating, as shown in Fig. 5(c). In particular, when the duty ratio is below 0.5 , the coupling of the graphene ribbons is negligible that the resonant wavelength of LSPs is identical to the case of isolated ribbon. ${ }^{92)}$ The resonant wavelength of LSPs approximately satisfied

$$
\lambda_{p}=2 \pi / \operatorname{Re}(\beta) \approx 2 w /(2 m+1),
$$

where $w$ is the width of ribbon, $m$ is the number of nodes with zero fields. Substituting Eq. (6) into Eq. (7), we can conclude the resonant wavelength is related to the ribbon width and the carrier concentration following the scaling behavior of $\lambda_{p} \propto w^{1 / 2}\left|\sigma_{s}\right|^{-1 / 2} \propto w^{1 / 2}\left|E_{F}\right|^{-1 / 2} \propto w^{1 / 2} n^{-1 / 4}$ (Fig. 5(d) and $5(\mathrm{e})$ ), which is different from the conventional twodimensional electron gas (2DEG), where $\lambda_{p} \propto w^{1 / 2} n^{-1 / 2}$. Note that the LSPs can be further influenced by the shape of particles, the distance between particles and the number of layers.

However, in the monolayer graphene, LSPs resonance depending on the carrier concentration is usually very weak, which leads to a limited modulation depth of frequency and strength $(\Delta A \sim 10 \%)$ by varying the Fermi level of graphene. Recently, the multilayer graphene/insulator structures ${ }^{37)}$ shown in Fig. 5(f), demonstrates a larger tunability. The absorption strength is greatly enhanced $(\Delta A \sim$ $50 \%$ ) and the resonant frequency is blue-shift with the increasing number of graphene disk (Fig. 5(g)). These phenomena are similar to the case of the monolayer graphene with the rise of carrier concentration or Fermi level. For the 
(a)

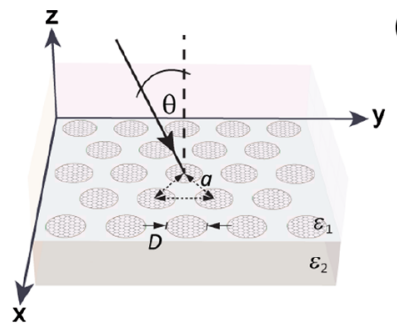

(b)

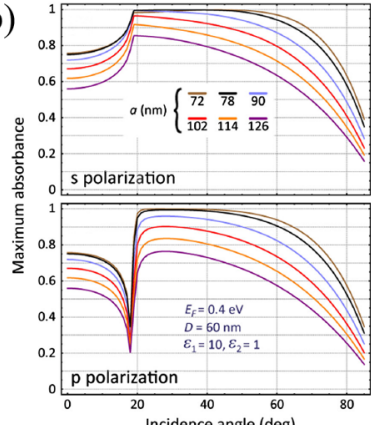

(c)

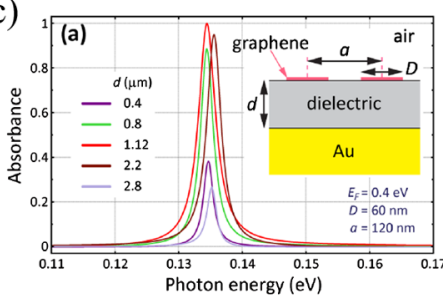

(d)

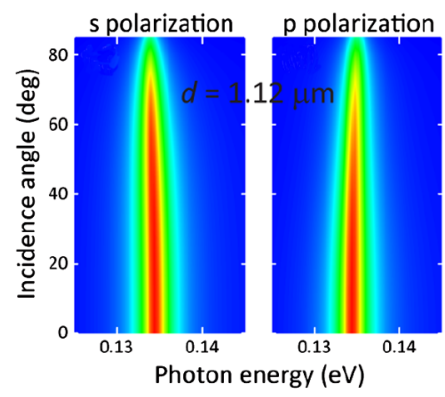

(e)

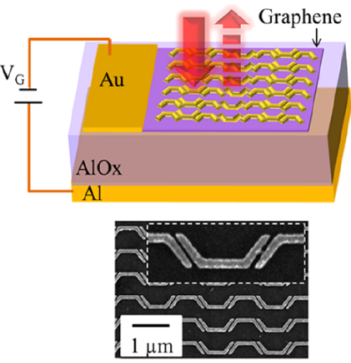

(f)

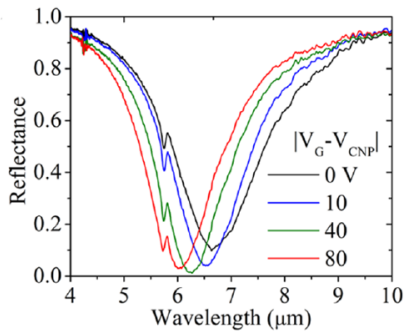

Fig. 6. (a) Schematic of the structured graphene sheet at the flat surface between two dielectrics. (b) Angular dependence of the peak absorbance for s- and p-polarization in arrays of graphene nano-disks. (c) Normal-incidence absorbance by graphene disk arrays supported on a dielectric-coated gold surface (inset) for various values of the dielectric film thickness. (d) Incidence-angle and photon-energy dependence of the absorbance under the conditions of (a) for $d=1.12 \mu \mathrm{m}$. (e) The schematic of a tunable metasurface absorber composed of an aluminum film, aluminum oxide layer, and a tunable metasurface on graphene. (f) Measured reflection spectra from the metasurface absorber for different gate voltages. (a) (d) used with permission from ${ }^{94)}$ copyright 2012 by American Physical Society. (e) and (f) used with permission from ${ }^{65}$ ) copyright 2014 by American Chemical Society.

multilayer graphene/insulator stacks, the resonant wavelength of LSPs needs to be taken the layer number into consideration and scaled as $\lambda_{p} \propto N^{-1 / 2}$ (Fig. 5(h)), leading to a relatively large tunable range compared with the monolayer graphene. In addition, the resonant wavelength of LSPs is significantly influenced by the Coulomb interactions among graphene disks, as shown in Fig. 5(i).

Although the multilayer graphene structure can realize a relatively large tunable range, the complicated preparation method limits the development of particle applications. Therefore, we will focus on the applications using the monolayer structured graphene in the following of this section.

\subsection{Modulation depth of graphene absorption}

A simple situation is first considered where the incident wave at an angle $\theta_{1}$ moves from a medium of refractive index $n_{1}$ to a second medium of refractive index $n_{2}$. A monolayer graphene with the surface conductivity of $\sigma_{s}$ at the interface between two dielectrics is treated as a zero thickness conductive film. The transmission and reflection coefficients for TE and TM polarized waves can be expressed by the modified Fresnel equations: ${ }^{93}$

$$
\begin{aligned}
t_{T E} & =\frac{2 \cos \theta_{1} n_{1}}{\cos \theta_{1} n_{1}+\cos \theta_{2} n_{2}+Z_{0} \sigma_{s}} \\
r_{T E} & =\frac{\cos \theta_{1} n_{1}-\cos \theta_{2} n_{2}-Z_{0} \sigma_{s}}{\cos \theta_{1} n_{1}+\cos \theta_{2} n_{2}+Z_{0} \sigma_{s}} \\
t_{T M} & =\frac{2 \cos \theta_{1} n_{1}}{\cos \theta_{2} n_{1}+\cos \theta_{1} n_{2}+\cos \theta_{1} \cos \theta_{2} Z_{0} \sigma_{s}}
\end{aligned}
$$

$$
r_{T M}=\frac{\cos \theta_{2} n_{1}-\cos \theta_{1} n_{2}-\cos \theta_{1} \cos \theta_{2} Z_{0} \sigma_{s}}{\cos \theta_{2} n_{1}+\cos \theta_{1} n_{2}+\cos \theta_{1} \cos \theta_{2} Z_{0} \sigma_{s}}
$$

where $Z_{0}=120 \pi$ is the wave impedance of vacuum, $\theta_{2}=$ $\operatorname{asin}\left(\sin \theta_{1} n_{1} / n_{2}\right)$ is the refractive angle. As a result, we get the power transmittance $\left(T=\frac{n_{2} \cos \theta_{2}}{n_{1} \cos \theta_{1}}|t|^{2}\right)$, reflectance $(R=$ $\left.|r|^{2}\right)$ and absorbance $(A=1-T-R)$. The absorbance $A$ is a function of surface conductivity $\left(\operatorname{Re}\left(\sigma_{s}\right)\right.$ and $\left.\operatorname{Im}\left(\sigma_{s}\right)\right)$. Based on the conditions of the extreme value for two variable functions, the maximum absorbances for TE and TM polarizations are ${ }^{94)}$

$$
\begin{aligned}
& A_{T E}^{\max }=\frac{1}{1+\operatorname{Re}(f)}, \\
& A_{T M}^{\max }=\frac{\left(\varepsilon_{1} / \varepsilon_{2}\right)|f|^{2}}{\operatorname{Re}(f)+\left(\varepsilon_{1} / \varepsilon_{2}\right)|f|^{2}} .
\end{aligned}
$$

where $f=\sqrt{\left(\varepsilon_{2} / \varepsilon_{1}\right)-\sin \theta_{1}^{2}} / \cos \theta_{1}$ and $\varepsilon_{1,2}=n_{1,2}^{2}$ is the relative permittivity. In the case of the normal incidence, Eqs. (12) and (13) are the same and equal to

$$
A_{\text {max }}=1 /\left(1+n_{2} / n_{1}\right),
$$

when the imaginary part of conductivity $\operatorname{Im}\left(\sigma_{s}\right)=0$ and the real part $\operatorname{Re}\left(\sigma_{s}\right)=\left(n_{1}+n_{2}\right) / Z_{0}$ are satisfied simultaneously. From Eqs. (12) and (13), it is possible to achieve a perfect absorption, when the incident angle is larger than the critical angle $\left(\theta_{1}>\operatorname{asin}\left(n_{2} / n_{1}\right), n_{1}>n_{2}\right)$, where the transmission wave is suppressed and converted into the evanescent wave 
absorbed by the graphene. Note that the maximum of absorbance is just a theoretical upper limit which does not contains any clues about how to structure the materials or whether some material is possible to achieve it. For example, there is an upper limit absorbance of $50 \%$ (100\% for coherent absorption ${ }^{95}$ ) under the normal incidence with the symmetric configuration (i.e. $n_{1}=n_{2}=1.0$ ). ${ }^{96,97)}$ However, it is impossible for the continuous graphene with $A_{\max }=50 \%$ in terahertz and infrared frequencies because the requirement of the surface conductivity including the real and imaginary parts cannot be satisfied simultaneously.

Alternatively, the structured graphene (ribbons, disks) or graphene/metasurface has more freedom degrees to tailor the surface conductivity, facilitating to realize the theoretical upper limit (Fig. 6(a)). ${ }^{94)}$ The effective surface conductivity can be extracted by the $S_{21}$ parameters from the numerical calculations

$$
\begin{aligned}
& \sigma_{s}^{\text {eff }} Z_{0}=\frac{2 \sqrt{n_{1} n_{2} \cos \theta_{1} \cos \theta_{2}}}{S_{21}}-\left(n_{1} \cos \theta_{1}+n_{2} \cos \theta_{2}\right) \\
& \text { (TE polarization) } \\
& \sigma_{s}^{\text {eff }} Z_{0}=\frac{2 \sqrt{n_{1} n_{2}}}{S_{21} \sqrt{\cos \theta_{1} \cos \theta_{2}}}-\frac{n_{1}}{\cos \theta_{1}}-\frac{n_{2}}{\cos \theta_{2}}
\end{aligned}
$$$$
\text { (TM polarization). }
$$

where $S_{21}$ parameter defined between two ports equals to $t \sqrt{n_{2} \cos \theta_{2}} / \sqrt{n_{1} \cos \theta_{1}}$. However, the theoretical study shows that the structured graphene only reach an absorption maximum in the limited incident angle due to the critical coupling (Fig. 6(b)). ${ }^{98)}$ Besides, the light transferred from the vacuum to medium $n_{1}$ undergoes a reflection loss in the actual situation that it is not a real perfect absorption.

To obtain the perfect absorption, the structured graphene or the graphene/metasurface on the top of a metallic ground plate separated by a thick dielectric spacer is adopted (Fig. 6(c) and (e)). ${ }^{65,99-101)}$ Based on the modified Fabry-Perot interference, we can get the total reflection coefficient as

$$
r=r_{1}-\frac{t_{1} t_{2} \exp (-2 i \varphi)}{1+r_{1} \exp (-2 i \varphi)}
$$

where $r_{1}$ and $t_{1}\left(t_{2}\right)$ are the reflection and transmission coefficients from medium $n_{1}\left(n_{2}\right)$ to $n_{2}\left(n_{1}\right)$ for TE or TM polarization, $\varphi=k_{0} n_{2} d / \cos \theta_{2}$ is the phase accumulation upon the propagation through dielectric. Due to the thick metallic plate in the back of dielectrics, there is no transmittance ( $T$ $=0$ ). Therefore, the absorbance is a function of the effective surface conductivity $\sigma_{s}^{e f f}$ and the phase accumulation $\varphi$. For example, the absorbance under the normal incidence reaches the maximum value when the conditions of $\operatorname{Re}\left(\sigma_{s}^{\text {eff }}\right)=n_{1} / Z_{0}$ and $\operatorname{Im}\left(\sigma_{s}^{\text {eff }}\right)=-n_{2} \cot (\varphi) / Z_{0}$ are satisfied simultaneously which can be easily realized by adjusting the thickness of dielectrics (Fig. 6(c)). ${ }^{101)}$ Importantly, the perfect absorber consisted of composite structure realizes an omnidirectional absorption (Fig. 6(d)) compared with the angle sensitive absorption from prism coupling (Fig. 6(b)). By varying the surface conductivity (or doping), the frequency and strength of the perfect absorber can be modulated in real time (Fig. $6(\mathrm{f}))^{65)}$

\subsection{Electromagnetic applications}

Except for the tunable ability of amplitude, the structured graphene is also capable to tune the phase and reshape the wavefront. For example, the graphene tailored into biaxial anisotropic nanoparticles can demonstrate different phase shifts along two principal axis due to the LSPs resonances. As a result, it can be utilized for polarization converter and optical activity. ${ }^{38-40)}$ Besides, the dynamic tunability of the effective surface conductivity by Fermi level further modulates the phase shift and amplitude for applications. For example, the asymmetric graphene nano-crosses (Fig. 7(a)) at Fermi level of $0.75 \mathrm{eV},{ }^{40)}$ demonstrate two distinct resonances with near $100^{\circ}$ phase abrupt under the normally incidence with polarizations along $\mathrm{x}$ - and $\mathrm{y}$-directions. At the wavelength of $7.92 \mu \mathrm{m}$, the transmission amplitude is equal and the phase difference approaches $90^{\circ}$ between the $\mathrm{x}$ and $\mathrm{y}$ components, indicating a quarter wave plate under the linearly polarized wave with a polarization angle of $45^{\circ}$. Besides, the transmission phase difference is always 0 at $7.85 \mu \mathrm{m}$ and $7.99 \mu \mathrm{m}$, indicating an optical activity. By varying the Fermi level, the structured graphene nano-crosses can realize a transition between a quarter wave plate and optical activity.

In addition, the structured graphene scatters with the desired phase (varying geometric parameters or Fermi levels) can be properly arranged to reshape the wavefront and realize many functionalities in ultrathin devices, such as flat lens, anomalous reflection and refraction. The study shows that the transmission phase almost realizes a full coverage by controlling the Fermi level of the graphene ribbons for a normally incident light polarized perpendicular to the nano-ribbons. ${ }^{41)}$ When two parallel graphene nano-ribbons are chosen as the unit cell, the transmission amplitude keeps uniform despite of the different Fermi levels. Hence, we can reshape the wavefront, i.e. anomalous refraction and lens (Fig. 7(b) and (c)) by arranging the distribution of nanoribbons with required Fermi levels. Besides, the structured graphene can be designed for the reflection modes ${ }^{42,43)}$ (Fig. 7(d) and (e)) or the circular polarized wave based on the Pancharatnam-Berry (P-B) phase ${ }^{44)}$ which is more suitable for broadband operation and multi-functions.

\section{Graphene/Metasurfaces}

\subsection{Graphene/metallic metasurfaces}

As mentioned above, the modulation depth of the graphene sheets, including frequency, amplitude and phase, usually exhibits a very limited tuning range due to the fact of a single atomic layer. One way to overcome this problem is using the structured graphene with the LSPs resonances in the $\mathrm{THz}$ and infrared frequencies, as mentioned above. Another effective solution is using the hybrid graphene/ metallic metasurfaces because the metasurfaces offer an 
(a)

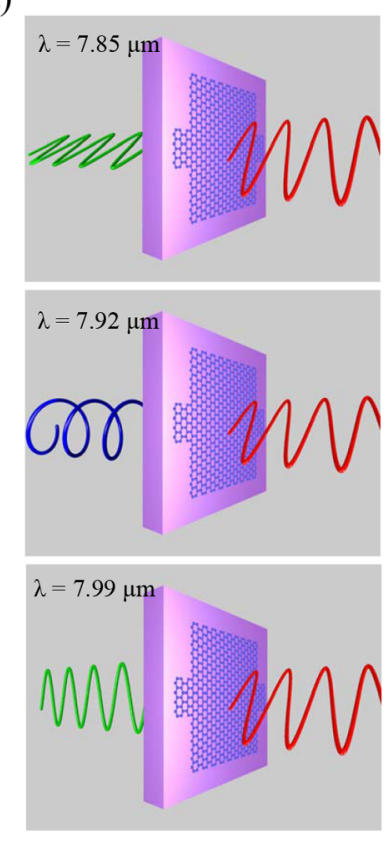

(b)

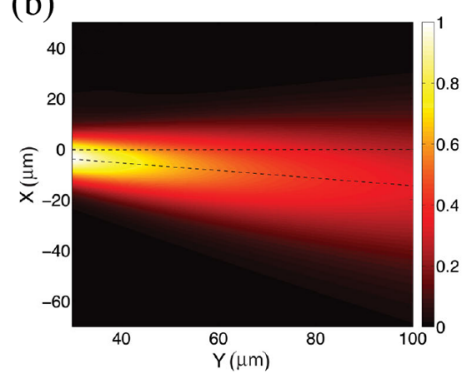

(d)

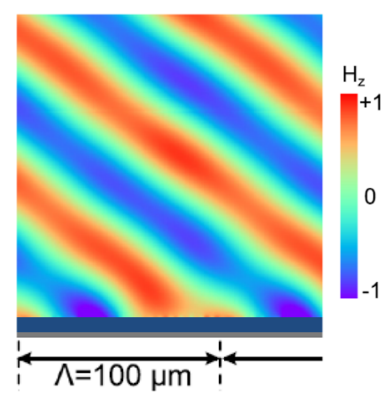

(c)

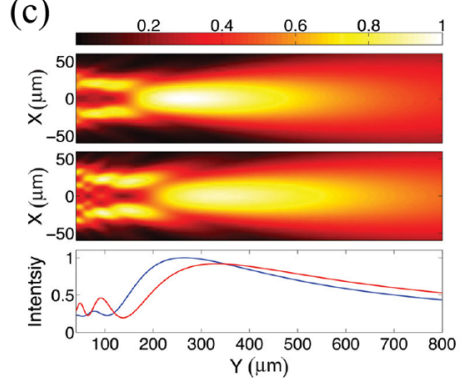

(e)

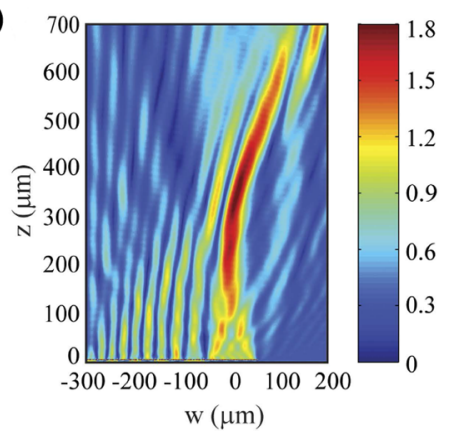

Fig. 7. (a) Graphene nano-crosses exhibit a giant optical activity (top \& bottom) or a quarter wave plate (middle) at different wavelengths. $(b, c)$ Anomalous refraction and flat lens composed of identical graphene bi-nanoribbon unit cells with different Fermi levels. (d) Anomalous reflection using graphene micro-ribbons/dielectric/metal with identical micro-ribbons but different Fermi levels and (e) An Airy beam generated by a graphene metasurface consisting of micro-ribbons/dielectric/ metal with identical Fermi levels but different widths of micro-ribbons. (a) used with permission from ${ }^{40}$ copyright 2013 by The Optical Society, (b) and (c) used with permission from ${ }^{41)}$ copyright 2014 by John Wiley and Sons, (d) used with permission from ${ }^{42)}$ copyright 2015 by AIP Publishing, (e) used with permission from ${ }^{43)}$ copyright 2015 by Nature Publishing.

enhanced light-mater interaction from the terahertz to the visible frequencies. The main drawback of the metasurfaces is that they are passive, i.e. the optical properties are fixed after fabrication. Therefore, the hybrid graphene/metasurface follows from their complementarity and demonstrates a more powerful application.

In the terahertz frequencies, the surface conductivity of graphene is mainly derived from the intra-band transitions. The imaginary part of the permittivity dominates the real part, that is, the intrinsic losses caused by the intra-band transitions play an important role in the optical properties of graphene. By varying Fermi level, the surface conductivity of graphene demonstrates a largely tunable range, facilitating to realize a deep amplitude modulation when it is combined with the metallic metasurface of strongly localized in-plane field. ${ }^{47,48,66,101-105)}$ Fig. 8(a) shows the metal gratings with a high duty ratio, where the localized electric fields in the slits are greatly enhanced (Fig. 8(b)) and the frequency is independent under the normal incidence with polarization perpendicular to the gratings. ${ }^{103)}$ The constructive interference between the coherent radiations from the oscillating local fields produces a high transmission. Therefore, the optical response of graphene/metallic gratings possess a broadband and deep modulation capacities (Fig. 8(c)). The change of transmission is mainly derived from the graphene absorption

$$
\Delta T=-\Delta A \sim S\left|E_{\text {ind }}\right|^{2} \eta^{2} \Delta \operatorname{Re}\left(\sigma_{s}\right)
$$

where $S$ is the graphene area, $E_{\text {inc }}$ is the electric field competent of incidence and $\eta=E / E_{\text {inc }}$ is the enhancement factor. From the Eq. (18), a large modulation depth can be obtained by greatly enhanced field despite of a little quality of surface conductivity. Beyond that, metallic resonators can exhibit strongly enhanced local fields for terahertz modulation by controlling the Fermi level of graphene, such as the splitring resonators (SRRs) with electric resonance, ${ }^{105)}$ the sandwich structure with magnetic resonance ${ }^{66}$ and the asymmetric structures with Fano resonance. ${ }^{47,104)}$

In the mid-infrared frequencies, the graphene behave as a plasmonic material with a low loss, that is, the real part of the graphene permittivity dominates over the imaginary part. The optical response of the graphene/plasmonic metasurface is mainly reflected in the change of the resonant frequency by varying the Fermi level of graphene. ${ }^{45,65,67,106-111)}$ Fig. 8(d) shows the asymmetric ח-shape plasmonic resonators which can exhibit high-sole asymmetric Fano resonances with a strong field enhancement when the incident polarization is parallel to the vertical resonators. ${ }^{109)}$ The Fano resonance is derived from the interference between a narrow resonance (dark mode, quadrupole resonance) and a broad continuum of states (bright mode, dipole resonance), leading to an enhanced transmission and a reduced reflec- 
(a)

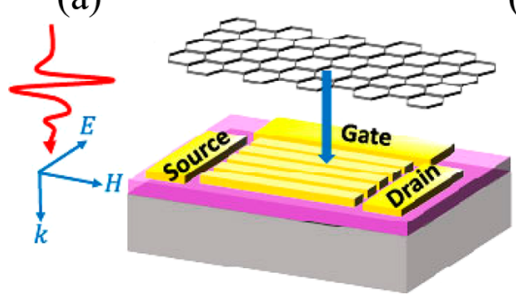

(d)

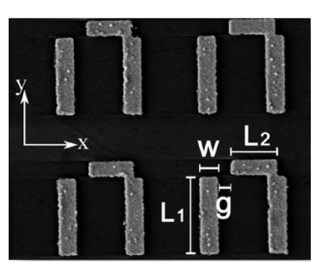

(g)

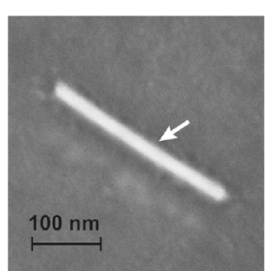

(b)

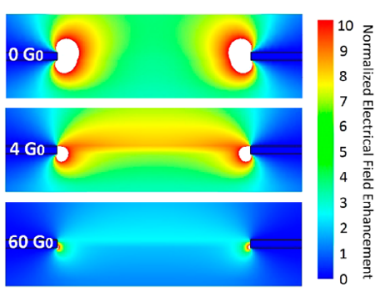

(e)

(h)
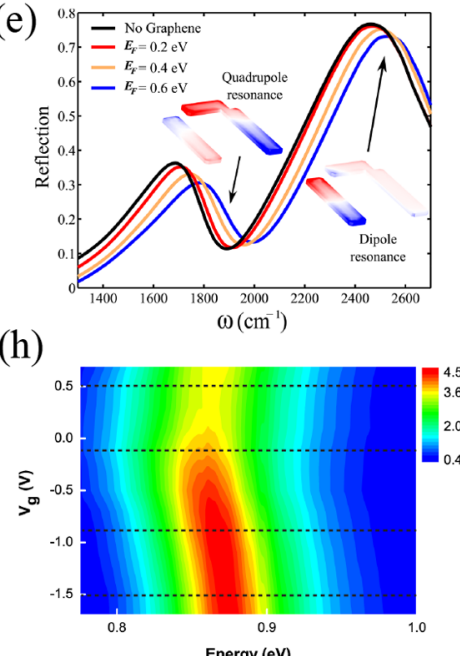

(c)

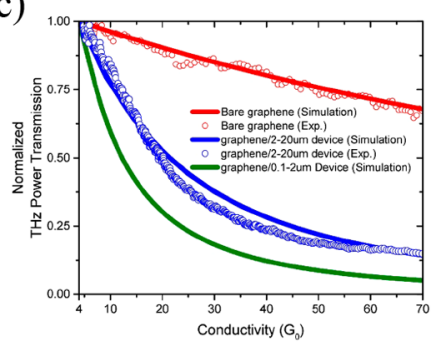

(f)

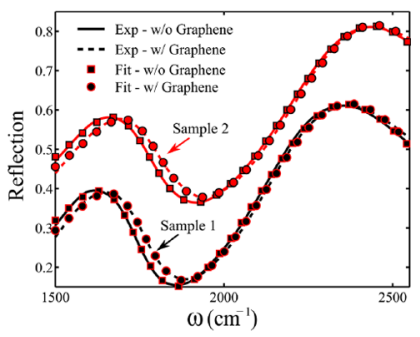

(i)

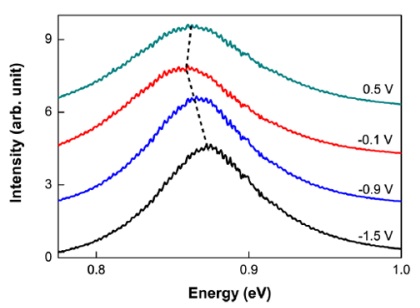

Fig. 8. (a) Hybrid graphene/metasurface device by transferring a single layer graphene on top of a gold slit device. The incoming $\mathrm{THz}$ wave is polarized perpendicular to the slit orientation. (b) Electrical field enhancement factor in the slit for different surface conductivity of graphene. (c) Simulated (solid lines) and experimental (empty cycles) results of normalized THz power transmission modulation for bare graphene and the hybrid graphene-metasurface structure as a function of graphene conductivity at $1 \mathrm{THz}$. (d) SEM image of the fabricated Fano-resonant metasurface composed of asymmetric חshape plasmonic resonators. (e) Simulated reflectivity spectra of hybrid graphene/metasurface for different Fermi levels. (f) Experimentally measured reflectivity spectra for the two samples with different geometrical dimensions before (solid curves) and after (dashed curves) graphene transfer. (g) A typical high resolution SEM image of the single gold nano-rod covered by graphene. (h) Scattering intensity (2D plot) is plotted as a function of the photon energy and gate voltage. (i) Four line cuts of the 2D plot for Rayleigh scattering spectra of an individual graphene-nano-rod hybrid structure. (a) - (i) used with permission from ${ }^{103,109,74)}$ copyright 2015, 2013, 2012 by American Chemical Society.

tion. ${ }^{112,113)}$ As shown in Fig. 8(e) and (f), the introduction of graphene to the metasurface significantly changes the frequency of Fano resonance, which can be evaluated by the perturbation theory and expressed as $^{75,109)}$

$$
\Delta \omega=\frac{\int_{S} \Delta \sigma_{s}^{i m a g}\left|E_{t}\right|^{2} d s}{W_{0}},
$$

where $W_{0}=2 \varepsilon_{0} \int_{V} \varepsilon|E|^{2} d v$ is the stored energy of the given mode. In the mid-infrared frequencies, the surface conductivity has a largely tunable range, endowing the broadband modulation of resonant frequency and resulting in a deep modulation of amplitude or phase at a certain frequency which can be used for optical switch, modulator and photonic applications.

In the near-infrared frequencies, the inter-band transitions become the decisive factor and give rise to considerable losses which can be utilized for the amplitude modulation. As shown in Fig. 1(a), the surface conductivity of graphene has a maximum of $e^{2} / 4 \hbar$ and produces a considerable losses of $2.3 \%$ at near-infrared frequencies. The limited varying range of the surface conductivity for isolated graphene sheet constrains the modulation depth. Therefore, the metasurfaces which offer a remarkable local field enhancement to the critical coupling with the tunable graphene are proposed. Fig. 8(g) shows a hybrid graphene/gold nano-rod device which can be efficiently controlled by the Fermi level due to the strongly confined the electric field in the vicinity of surface at the LSPs resonance. ${ }^{74)}$ The Rayleigh scattering spectra, demonstrate the efficient electrical modulation of plasmon resonance $(6 \%)$ at near infrared frequency utilizing the strong and tunable inter-band transitions in graphene (Fig. 8(h) and (i)). The change of amplitude can be obtained by $^{75,114)}$

$$
\Delta T \approx-\Delta A=\frac{-\Delta \sigma_{s}^{\text {real }} \int_{S} E_{t}^{2} d s}{c_{0} \varepsilon_{0}\left|E_{\text {ind }}\right|^{2} A},
$$


where $c_{0}$ is the speed of light, $\varepsilon_{0}$ is the vacuum permittivity, $A$ is the surface area of the unit cell, $\left|E_{t}\right|=\eta\left|E_{\text {inc }}\right|$ is the tangent electric field in the graphene with an enhancement factor $\eta$ compared with the incident electric $E_{\text {inc. }}$. To further improve the modulation depth in the near infrared frequency, the graphene should interact with high quality factor ( $Q$-factor) resonances, such as the Fano resonances emerged in the asymmetric structures or plasmonic clusters. ${ }^{46,68)}$ Note that high $Q$-factor resonance usually accompany with a narrow bandwidth which demands the nearinfrared detector with a high resolution in reverse.

\subsection{Graphene/dielectric metasurfaces}

In the above content, we mainly focus on the hybrid graphene/metallic metasurfaces. In the near-infrared and optical frequencies, however, the non-radiative losses in metal lead to a low $Q$-factor resonance and a limited modulation depth. ${ }^{114-116)}$ Alternatively, the dielectric metasurfaces with high refractive index dielectric particles embedded in a low index matrix can support different order electric and magnetic resonances with an extremely low intrinsic losses based on the Mie theory. ${ }^{61-63)}$ For example, the asymmetric structures, such as asymmetric Si-based II-shape and $\pi$ shape resonators, ${ }^{117,118)}$ demonstrate a high $Q$-factor Fano resonances and a strongly localized field enhancement in the gaps, facilitating to the depth modulation by varying the Fermi level (Fig. 9(a)). Similarly, the asymmetric periodity also has the same functions as the asymmetric structure. ${ }^{75)}$ Fig. 9(b) shows an 85\% modulation depth for the lefthanded passband with $2 \pi$ phase coverage when the Fermi level is varied from 0 to $0.8 \mathrm{eV}$. The modulation depth may be further improved by decreasing the asymmetric degree, ${ }^{119)}$ but it also brings a challenge to a high machining precision and a higher Fermi level to realize high transmission, which makes the conventional gating configuration not suitable any more. Up to date, the experimental demonstration of dynamic tunability via graphene/dielectric metasurface has not yet been emerged.

Another solution to realize a broadband or deep modulation is using dielectric waveguide or photonic crystal ${ }^{16,120-124)}$ which supports the guided mode or optical cavity resonance with the electric field confined in vicinity of surface. Fig. 9(c) shows the graphene/dielectric waveguide device which exhibits a large modulation depth of $0.1 \mathrm{~dB}_{\mu \mathrm{m}}^{-1}$ (Fig. 9(d)) and a broadband modulation range from $1.35 \mu \mathrm{m}$ to $1.6 \mu \mathrm{m} .{ }^{16)}$ By introducing defects in the photonic crystal, ${ }^{123)}$ as shown in Fig. 9(e), the localized field are greatly enhanced near the region of defects. The hybrid graphene/2D photonic crystal demonstrate a $6 \mathrm{~dB}$ modulation for the cavity resonance with $1.5 \mathrm{~nm}$ line width in reflectivity, as shown in Fig. 9(f).

In addition to the above mentioned applications of optical modulator or switch, the graphene integrated with nanoparticles with the greatly enhanced local fields are also promising applications for the optical bistability, ${ }^{125,126)}$ superscattering effect ${ }^{127-129)}$ and enhanced photo-detection. ${ }^{130,131)}$

\section{Conclusions and Outlook}

Graphene combines many superior properties from mechanical to electronic, has attracted intensive research interests from different fields. Owning to the high carrier mobility and tunable carrier concentration, graphene opens up an (a)

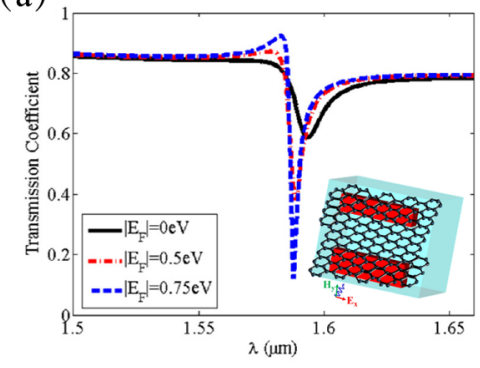

(c)

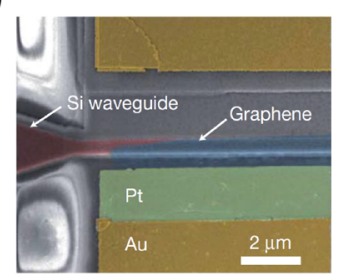

(d) (b)
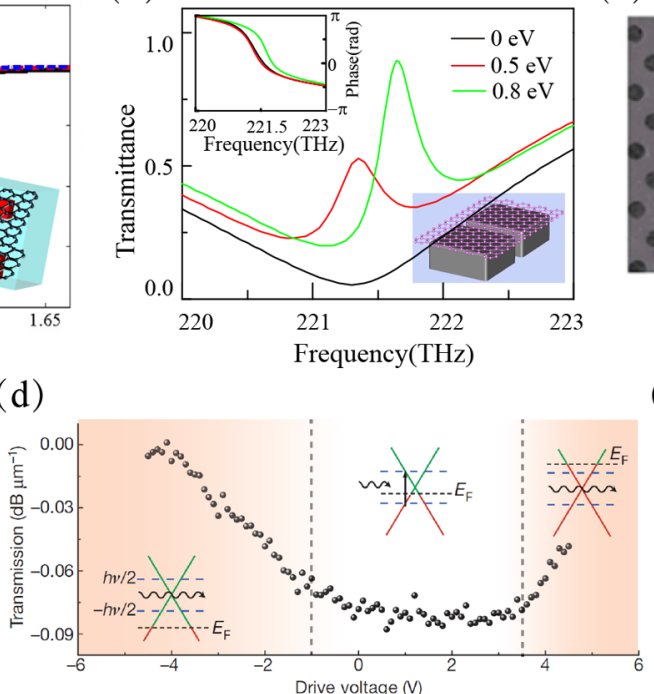

(e)

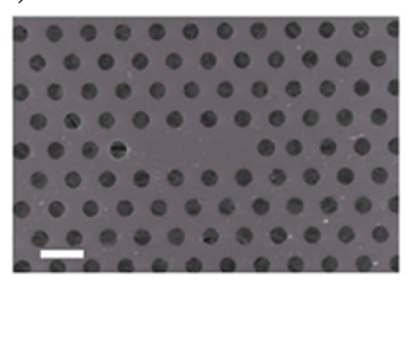

(f)

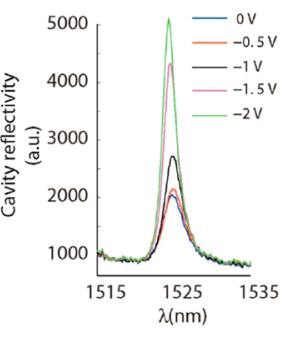

Fig. 9. (a, b) Transmission spectra from the hybrid graphene/dielectric metasurface consisted of II-shape dielectric bars with structure asymmetry or dielectric blocks with periodity asymmetry for different Fermi levels. (c) SEM image of the hybrid graphene/dielectric waveguide modulator. (d) Static electro-optical response of the device for different gate voltages at $1.53 \mu \mathrm{m}$. (e) SEM image of the hybrid graphene/photonic crystal device. (f) Measured reflectivity from the hybrid device for different gate voltages. (a) used with permission from ${ }^{117}$ copyright 2015 by The Optical Society, (c) and (d) used with permission from ${ }^{16)}$ copyright 2011 by Nature Publishing, (e) and (f) used with permission from ${ }^{123)}$ copyright 2013 by American Chemical Society. 
exciting opportunity to high performance applications, such as plasmonics, optoelectronic devices based on graphene plasmons. Different from noble metal plasmons in range of visible frequencies, graphene plasmons operate from terahertz to mid-infrared frequencies and demonstrate an extremely high confinement, facilitating to light-matter interaction for quantum optics, chemical identifications and biosensors. Importantly, the tunable surface conductivity makes graphene a wider application prospect. Graphene integrated with silicon-based optical circuit can dynamically manipulate the amplitude, phase and polarization of light, promising applications for optical communication and optical computing. Besides, graphene can couple with metasurfaces of enhanced local electric field to improve the optical modulation depth.

The behavior of graphene plasmons is tightly associated with the graphene itself. Large-scale grown high quality graphene is desirable in many applications and has been realized by chemical vapor deposition (CVD) on copper foils and films. However, the following transfer process is usually very complicated and need be optimized to minimize the damage to graphene. Another important challenge is the fabrication of structured graphene with fine control of shape and quality which will significantly influence the graphene plasmons.

Note that except for the graphene, many $2 \mathrm{D}$ materials, such as black phosphorus, molybdenum disulfide and topological insulators, also demonstrate the similar or even preferable electrical and optical properties which can be used to explore the tunable collective optical oscillations.

\section{Acknowledgments}

This work was supported by grants from the National Youth Top-Notch Talent Support Program and the Fundamental Research Funds for the Central Universities.

\section{REFERENCES}

1. K. S. Novoselov, A. K. Geim, S. V. Morozov, D. Jiang, Y. Zhang, S. V. Dubonos, I. V. Grigorieva, and A. A. Firsov, "Electric Field Effect in Atomically Thin Carbon Films," Science, 306 [5696] 666-69 (2004).

2. A. C. Neto, F. Guinea, N. M. Peres, K. S. Novoselov, and A. K. Geim, "The Electronic Properties of Graphene," Rev. Mod. Phys., 81 [1] 109 (2009).

3. L. Falkovsky, "Optical Properties of Graphene," J. Phys.: Conf. Ser., 129 [1] 012004 (2008).

4. M. C. Lemme, T. J. Echtermeyer, M. Baus, and H. Kurz, "A Graphene Field-Effect Device," IEEE Electron Device Lett., 28 [4] 282-84 (2007).

5. F. Bonaccorso, Z. Sun, T. Hasan, and A. Ferrari, "Graphene Photonics and Optoelectronics," Nat. Photonics, 4 [9] 61122 (2010).

6. C. H. Lui, K. F. Mak, J. Shan, and T. F. Heinz, "Ultrafast Photoluminescence from Graphene," Phys. Rev. Lett., 105 [12] 127404 (2010).
7. J. M. Dawlaty, S. Shivaraman, J. Strait, P. George, M. Chandrashekhar, F. Rana, M. G. Spencer, D. Veksler, and Y. Chen, "Measurement of the Optical Absorption Spectra of Epitaxial Graphene from Terahertz to Visible," Appl. Phys. Lett., 93 [13] 131905 (2008).

8. L. Falkovsky and S. Pershoguba, "Optical Far-Infrared Properties of a Graphene Monolayer and Multilayer," Phys. Rev. B, 76 [15] 153410 (2007).

9. R. R. Nair, P. Blake, A. N. Grigorenko, K. S. Novoselov, T. J. Booth, T. Stauber, N. M. Peres, and A. K. Geim, "Fine Structure Constant Defines Visual Transparency of Graphene," Science, 320 [5881] 1308 (2008).

10. M. Jablan, H. Buljan, and M. Soljačić, "Plasmonics in Graphene at Infrared Frequencies," Phys. Rev. B, 80 [24] 245435 (2009).

11. A. Grigorenko, M. Polini, and K. Novoselov, "Graphene Plasmonics," Nat. Photonics, 6 [11] 749-58 (2012).

12. F. H. Koppens, D. E. Chang, and F. J. García de Abajo, "Graphene Plasmonics: A Platform for Strong LightMatter Interactions," Nano Lett., 11 [8] 3370-77 (2011).

13. J. Chen, M. Badioli, P. Alonso-González, S. Thongrattanasiri, F. Huth, J. Osmond, M. Spasenović, A. Centeno, A. Pesquera, and P. Godignon, "Optical Nano-Imaging of Gate-Tunable Graphene Plasmons,” Nature, 487 [7405] 77-81 (2012).

14. A. Kasry, M. A. Kuroda, G. J. Martyna, G. S. Tulevski, and A. A. Bol, "Chemical Doping of Large-Area Stacked Graphene Films for Use as Transparent, Conducting Electrodes," ACS Nano., 4 [7] 3839-44 (2010).

15. F. Wang, Y. Zhang, C. Tian, C. Girit, A. Zettl, M. Crommie, and Y. R. Shen, "Gate-Variable Optical Transitions in Graphene," Science, 320 [5873] 206-9 (2008).

16. M. Liu, X. Yin, E. Ulin-Avila, B. Geng, T. Zentgraf, L. Ju, F. Wang, and X. Zhang, "A Graphene-based Broadband Optical Modulator," Nature, 474 [7349] 64-7 (2011).

17. A. Y. Nikitin, F. Guinea, F. Garcia-Vidal, and L. MartinMoreno, "Fields Radiated by a Nanoemitter in a Graphene Sheet," Phys. Rev. B, 84 [19] 195446 (2011).

18. Y. Bao, S. Zu, Y. Zhang, and Z. Fang, "Active Control of Graphene-based Unidirectional Surface Plasmon Launcher," ACS Photonics, 2 [8] 1135-40 (2015).

19. Z. Fei, A. S. Rodin, G. O. Andreev, W. Bao, A. S. McLeod, M. Wagner, L. M. Zhang, Z. Zhao, M. Thiemens, G. Dominguez, M. M. Fogler, A. H. C. Neto, C. N. Lau, F. Keilmann, and D. N. Basov, "Gate-Tuning of Graphene Plasmons Revealed by Infrared Nano-Imaging," Nature, 487 [7405] 82-5 (2012).

20. J. A. Gerber, S. Berweger, B. T. O'Callahan, and M. B. Raschke, "Phase-Resolved Surface Plasmon Interferometry of Graphene," Phys. Rev. Lett., 113 [5] 055502 (2014).

21. G. X. Ni, L. Wang, M. D. Goldflam, M. Wagner, Z. Fei, A. S. McLeod, M. K. Liu, F. Keilmann, B. Özyilmaz, A. H. Castro Neto, J. Hone, M. M. Fogler, and D. N. Basov, "Ultrafast Optical Switching of Infrared Plasmon Polaritons in High-Mobility Graphene," Nat. Photonics, 10 [4] 244-47 (2016).

22. W. Zhou, J. Lee, J. Nanda, S. T. Pantelides, S. J. Pennycook, and J.-C. Idrobo, "Atomically Localized Plasmon Enhancement in Monolayer Graphene," Nat. Nanotech- 
nol., 7 [3] 161-65 (2012).

23. Z. Fei, A. S. Rodin, W. Gannett, S. Dai, W. Regan, M. Wagner, M. K. Liu, A. S. McLeod, G. Dominguez, M. Thiemens, Antonio H. Castro Neto, F. Keilmann, A. Zettl, R. Hillenbrand, M. M. Fogler, and D. N. Basov, "Electronic and Plasmonic Phenomena at Graphene Grain Boundaries," Nat. Nanotechnol., 8 [11] 821-25 (2013).

24. W. Gao, G. Shi, Z. Jin, J. Shu, Q. Zhang, R. Vajtai, P. M. Ajayan, J. Kono, and Q. Xu, "Excitation and Active Control of Propagating Surface Plasmon Polaritons in Graphene," Nano Lett., 13 [8] 3698-702 (2013).

25. W. Gao, J. Shu, C. Qiu, and Q. Xu, "Excitation of Plasmonic Waves in Graphene by Guided-Mode Resonances," ACS Nano., 6 [9] 7806-13 (2012).

26. H. Lu, J. Zhao, and M. Gu, "Nanowires-Assisted Excitation and Propagation of Mid-Infrared Surface Plasmon Polaritons in Graphene," J. Appl. Phys., 120 [16] 163106 (2016).

27. Y. Zhao, G. Chen, Z. Tao, C. Zhang, and Y. Zhu, "High QFactor Plasmonic Resonators in Continuous Graphene Excited by Insulator-Covered Silicon Gratings," RSC $A d v ., 4$ [50] 26535-42 (2014).

28. Y. V. Bludov, M. Vasilevskiy, and N. Peres, "Mechanism for Graphene-based Optoelectronic Switches by Tuning Surface Plasmon-Polaritons in Monolayer Graphene," Europhys. Lett., 92 [6] 68001 (2011).

29. A. Y. Nikitin, P. Alonso-González, and R. Hillenbrand, "Efficient Coupling of Light to Graphene Plasmons by Compressing Surface Polaritons with Tapered Bulk Materials," Nano Lett., 14 [5] 2896-901 (2014).

30. V. W. Brar, M. S. Jang, M. Sherrott, J. J. Lopez, and H. A. Atwater, "Highly Confined Tunable Mid-Infrared Plasmonics in Graphene Nanoresonators," Nano Lett., 13 [6] 2541-47 (2013).

31. H. Hu, X. Yang, F. Zhai, D. Hu, R. Liu, K. Liu, Z. Sun, and Q. Dai, "Far-Field Nanoscale Infrared Spectroscopy of Vibrational Fingerprints of Molecules with Graphene Plasmons," Nat. Commun., 712334 (2016).

32. L. Ju, B. Geng, J. Horng, C. Girit, M. Martin, Z. Hao, H. A. Bechtel, X. Liang, A. Zettl, and Y. R. Shen, "Graphene Plasmonics for Tunable Terahertz Metamaterials," Nat. Nanotechnol., 6 [10] 630-34 (2011).

33. H. Yan, T. Low, W. Zhu, Y. Wu, M. Freitag, X. Li, F. Guinea, P. Avouris, and F. Xia, "Damping Pathways of MidInfrared Plasmons in Graphene Nanostructures," Nat. Photonics, 7 [5] 394-99 (2013).

34. D. Rodrigo, O. Limaj, D. Janner, D. Etezadi, F. J. G. de Abajo, V. Pruneri, and H. Altug, "Mid-Infrared Plasmonic Biosensing with Graphene," Science, 349 [6244] 165-68 (2015).

35. F. J. Garcia de Abajo, "Graphene Plasmonics: Challenges and Opportunities," ACS Photonics, 1 [3] 135-52 (2014).

36. Z. Fang, S. Thongrattanasiri, A. Schlather, Z. Liu, L. Ma, Y. Wang, P. M. Ajayan, P. Nordlander, N. J. Halas, and F. J. García de Abajo, "Gated Tunability and Hybridization of Localized Plasmons in Nanostructured Graphene," ACS Nano., 7 [3] 2388-95 (2013).

37. H. Yan, X. Li, B. Chandra, G. Tulevski, Y. Wu, M. Freitag, W. Zhu, P. Avouris, and F. Xia, "Tunable Infrared Plasmonic Devices Using Graphene/Insulator Stacks," Nat. Nano- technol., 7 [5] 330-34 (2012).

38. T. Guo and C. Argyropoulos, "Broadband Polarizers based on Graphene Metasurfaces," Opt. Lett., 41 [23] 5592-95 (2016).

39. A. Fallahi and J. Perruisseau-Carrier, "Design of Tunable Biperiodic Graphene Metasurfaces,” Phys. Rev. B, 86 [19] 195408 (2012).

40. H. Cheng, S. Chen, P. Yu, J. Li, L. Deng, and J. Tian, "Mid-Infrared Tunable Optical Polarization Converter Composed of Asymmetric Graphene Nanocrosses," Opt. Lett., 38 [9] 1567-69 (2013).

41. F. Lu, B. Liu, and S. Shen, "Infrared Wavefront Control based on Graphene Metasurfaces," Adv. Opt. Mater., 2 [8] 794-99 (2014).

42. T. Yatooshi, A. Ishikawa, and K. Tsuruta, "Terahertz Wavefront Control by Tunable Metasurface Made of Graphene Ribbons," Appl. Phys. Lett., 107 [5] 053105 (2015).

43. Z. Li, K. Yao, F. Xia, S. Shen, J. Tian, and Y. Liu, "Graphene Plasmonic Metasurfaces to Steer Infrared Light," Sci. Rep., 512423 (2015).

44. X. Hu, L. Wen, S. Song, and Q. Chen, "Tunable Graphene Metasurfaces by Discontinuous Pancharatnam-Berry Phase Shift," Nanotechnology, 26 [50] 505203 (2015).

45. N. Dabidian, I. Kholmanov, A. B. Khanikaev, K. Tatar, S. Trendafilov, S. H. Mousavi, C. Magnuson, R. S. Ruoff, and G. Shvets, "Electrical Switching of Infrared Light Using Graphene Integration with Plasmonic Fano Resonant Metasurfaces," ACS Photonics, 2 [2] 216-27 (2015).

46. N. K. Emani, T.-F. Chung, A. V. Kildishev, V. M. Shalaev, Y. P. Chen, and A. Boltasseva, "Electrical Modulation of Fano Resonance in Plasmonic Nanostructures Using Graphene," Nano Lett., 14 [1] 78-82 (2013).

47. S. H. Lee, M. Choi, T.-T. Kim, S. Lee, M. Liu, X. Yin, H. K. Choi, S. S. Lee, C.-G. Choi, and S.-Y. Choi, "Switching Terahertz Waves with Gate-Controlled Active Graphene Metamaterials," Nat. Mater., 11 [11] 936-41 (2012).

48. M. M. Jadidi, A. B. Sushkov, R. L. Myers-Ward, A. K. Boyd, K. M. Daniels, D. K. Gaskill, M. S. Fuhrer, H. D. Drew, and T. E. Murphy, "Tunable Terahertz Hybrid Metal-Graphene Plasmons," Nano Lett., 15 [10] 7099-104 (2015).

49. X. Ni, A. V. Kildishev, and V. M. Shalaev, "Metasurface Holograms for Visible Light," Nat. Commun., 42807 (2013).

50. G. Zheng, H. Mühlenbernd, M. Kenney, G. Li, T. Zentgraf, and S. Zhang, "Metasurface Holograms Reaching 80\% Efficiency," Nat. Nanotechnol., 10 [4] 308-12 (2015).

51. D. Wen, F. Yue, G. Li, G. Zheng, K. Chan, S. Chen, M. Chen, K. F. Li, P. W. H. Wong, K. W. Cheah, E. Yue Bun Pun, S. Zhang, and X. Chen, "Helicity Multiplexed Broadband Metasurface Holograms," Nat. Commun., 68241 (2015).

52. X. Ni, S. Ishii, A. V. Kildishev, and V. M. Shalaev, "UltraThin, Planar, Babinet-Inverted Plasmonic Metalenses," Light Sci. Appl., 2 [4] e72 (2013).

53. F. Aieta, P. Genevet, M. A. Kats, N. Yu, and R. Blanchard, "Aberration-free Ultrathin Flat Lenses and Axicons at Telecom Wavelengths based on Plasmonic Metasurfaces," Nano Lett., 12 4932-36 (2012).

54. M. Kang, T. Feng, H.-T. Wang, and J. Li, "Wave Front 
Engineering from an Array of Thin Aperture Antennas," Opt. Express, 20 [14] 15882-90 (2012).

55. C. Liu, Y. Bai, L. Jing, Y. Yang, H. Chen, J. Zhou, Q. Zhao, and L. Qiao, "Equivalent Energy Level Hybridization Approach for High-Performance Metamaterials Design," Acta Mater., 135 [15] 144-49 (2017).

56. N. Yu, P. Genevet, M. A. Kats, F. Aieta, J.-P. Tetienne, F. Capasso, and Z. Gaburro, "Light Propagation with Phase Discontinuities: Generalized Laws of Reflection and Refraction," Science, 334 [6054] 333-37 (2011).

57. C. Liu, Y. Bai, Q. Zhao, Y. Yang, H. Chen, J. Zhou, and L. Qiao, "Fully Controllable Pancharatnam-Berry Metasurface Array with High Conversion Efficiency and Broad Bandwidth," Sci. Rep., 634819 (2016).

58. X. Ni, N. K. Emani, A. V. Kildishev, A. Boltasseva, and V. M. Shalaev, "Broadband Light Bending with Plasmonic Nanoantennas," Science, 335 [6067] 427 (2012).

59. D. R. Smith, W. J. Padilla, D. C. Vier, S. C. Nemat-Nasser, and S. Schultz, "Composite Medium with Simultaneously Negative Permeability and Permittivity," Phys. Rev. Lett., 84 [18] 4184-87 (2000).

60. D. Schurig, J. J. Mock, B. J. Justice, S. A. Cummer, J. B. Pendry, A. F. Starr, and D. R. Smith, "Metamaterial Electromagnetic Cloak at Microwave Frequencies," Science, 314[5801] 977-80 (2006).

61. Y. Yang, W. Wang, P. Moitra, I. I. Kravchenko, D. P. Briggs, and J. Valentine, "Dielectric Meta-Reflectarray for Broadband Linear Polarization Conversion and Optical Vortex Generation," Nano Lett., 14 [3] 1394-99 (2014).

62. K. E. Chong, I. Staude, A. James, J. Dominguez, S. Liu, S. Campione, G. S. Subramania, T. S. Luk, M. Decker, and D. N. Neshev, "Polarization-Independent Silicon Metadevices for Efficient Optical Wavefront Control," Nano Lett., 15 [8] 5369-74 (2015).

63. M. I. Shalaev, J. Sun, A. Tsukernik, A. Pandey, K. Nikolskiy, and N. M. Litchinitser, "High-Efficiency All-Dielectric Metasurfaces for Ultracompact Beam Manipulation in Transmission Mode," Nano Lett., 15 [9] 6261-66 (2015).

64. W. Gao, J. Shu, K. Reichel, D. V. Nickel, X. He, G. Shi, R. Vajtai, P. M. Ajayan, J. Kono, and D. M. Mittleman, "HighContrast Terahertz Wave Modulation by Gated Graphene Enhanced by Extraordinary Transmission through Ring Apertures," Nano Lett., 14 [3] 1242-48 (2014).

65. Y. Yao, R. Shankar, M. A. Kats, Y. Song, J. Kong, M. Loncar, and F. Capasso, "Electrically Tunable Metasurface Perfect Absorbers for Ultrathin Mid-Infrared Optical Modulators," Nano Lett., 14 [11] 6526-32 (2014).

66. Z. Miao, Q. Wu, X. Li, Q. He, K. Ding, Z. An, Y. Zhang, and L. Zhou, "Widely Tunable Terahertz Phase Modulation with Gate-Controlled Graphene Metasurfaces," Phys. Rev. $X, 5$ [4] 041027 (2015).

67. N. Dabidian, S. Dutta-Gupta, I. Kholmanov, K. Lai, F. Lu, J. Lee, M. Jin, S. Trendafilov, A. Khanikaev, and B. Fallahazad, "Experimental Demonstration of Phase Modulation and Motion Sensing Using Graphene-Integrated Metasurfaces," Nano Lett., 16 [6] 3607-15 (2016).

68. Z. Fang, Z. Liu, Y. Wang, P. M. Ajayan, P. Nordlander, and N. J. Halas, "Graphene-Antenna Sandwich Photodetector," Nano Lett., 12 [7] 3808-13 (2012).
69. Y. Fan, N. H. Shen, F. Zhang, Z. Wei, H. Li, Q. Zhao, Q. Fu, P. Zhang, T. Koschny, and C. M. Soukoulis, "Electrically Tunable Goos-Hänchen Effect with Graphene in the Terahertz Regime," Adv. Opt. Mater., 4 [11] 1824-28 (2016).

70. K. S. Kim, Y. Zhao, H. Jang, S. Y. Lee, J. M. Kim, K. S. Kim, J.-H. Ahn, P. Kim, J.-Y. Choi, and B. H. Hong, "LargeScale Pattern Growth of Graphene Films for Stretchable Transparent Electrodes," Nature, 457 [7230] 706 (2009).

71. X. Li, C. W. Magnuson, A. Venugopal, R. M. Tromp, J. B. Hannon, E. M. Vogel, L. Colombo, and R. S. Ruoff, "LargeArea Graphene Single Crystals Grown by Low-Pressure Chemical Vapor Deposition of Methane on Copper," J. Am. Chem. Soc., 133 [9] 2816-19 (2011).

72. E. V. Castro, H. Ochoa, M. Katsnelson, R. Gorbachev, D. Elias, K. Novoselov, A. Geim, and F. Guinea, "Limits on Charge Carrier Mobility in Suspended Graphene due to Flexural Phonons," Phys. Rev. Lett., 105 [26] 266601 (2010).

73. C.-F. Chen, C.-H. Park, B. W. Boudouris, J. Horng, B. Geng, C. Girit, A. Zettl, M. F. Crommie, R. A. Segalman, S. G. Louie, and F. Wang, "Controlling Inelastic Light Scattering Quantum Pathways in Graphene," Nature, 471 [7340] 617-20 (2011).

74. J. Kim, H. Son, D. J. Cho, B. Geng, W. Regan, S. Shi, K. Kim, A. Zettl, Y.-R. Shen, and F. Wang, "Electrical Control of Optical Plasmon Resonance with Graphene," Nano Lett., 12 [11] 5598-602 (2012).

75. C. Liu, Y. Bai, J. Zhou, Q. Zhao, and L. Qiao, "Large-Scale Modulation of Left-Ganded Passband in Gybrid Graphene/ Dielectric Metasurface," Ann. Phys., 529 [8] 1700125 (2017).

76. Z. Fang, Y. Wang, Z. Liu, A. Schlather, P. M. Ajayan, F. H. Koppens, P. Nordlander, and N. J. Halas, "PlasmonInduced Doping of Graphene," ACS Nano., 6 [11] 1022228 (2012).

77. H. Yan, Z. Li, X. Li, W. Zhu, P. Avouris, and F. Xia, "Infrared Spectroscopy of Tunable Dirac Terahertz MagnetoPlasmons in Graphene," Nano Lett., 12 [7] 3766-71 (2012).

78. V. P. Verma, S. Das, I. Lahiri, and W. Choi, "Large-Area Graphene on Polymer Film for Flexible and Transparent Anode in Field Emission Device," Appl. Phys. Lett., 96 [20] 203108 (2010).

79. F. Schedin, E. Lidorikis, A. Lombardo, V. G. Kravets, A. K. Geim, A. N. Grigorenko, K. S. Novoselov, and A. C. Ferrari, "Surface-Enhanced Raman Spectroscopy of Graphene," ACS Nano., 4 [10] 5617-26 (2010).

80. S. Mikhailov and K. Ziegler, "New Electromagnetic Mode in Graphene," Phys. Rev. Lett., 99 [1] 016803 (2007).

81. Y. Liu, R. F. Willis, K. Emtsev, and T. Seyller, "Plasmon Dispersion and Damping in Electrically Isolated TwoDimensional Charge Sheets," Phys. Rev. B, 78 [20] 201403 (2008).

82. Z. Fei, G. O. Andreev, W. Bao, L. M. Zhang, A. S. McLeod, C. Wang, M. K. Stewart, Z. Zhao, G. Dominguez, and M. Thiemens, "Infrared Nanoscopy of Dirac Plasmons at the Graphene-SiO ${ }_{2}$ Interface," Nano Lett., 11 [11] 4701-5 (2011).

83. W. L. Barnes, A. Dereux, and T. W. Ebbesen, "Surface Plasmon Subwavelength Optics," Nature, 424 [6950] 824 (2003).

84. A. Vakil and N. Engheta, "Transformation Optics Using 
Graphene," Science, 332 [6035] 1291-94 (2011).

85. A. Otto, "Excitation of Nonradiative Surface Plasma Waves in Silver by the Method of Frustrated Total Reflection," $Z$. Phys., 216 [4] 398-410 (1968).

86. E. Kretschmann and H. Raether, "Radiative Decay of Non Radiative Surface Plasmons Excited by Light," Z. Naturforsch. A, 23 [12] 2135-36 (1968).

87. P. Alonso-González, A. Y. Nikitin, F. Golmar, A. Centeno, A. Pesquera, S. Vélez, J. Chen, G. Navickaite, F. Koppens, and A. Zurutuza, "Controlling Graphene Plasmons with Resonant Metal Antennas and Spatial Conductivity Patterns," Science, 344 [6190] 1369-73 (2014).

88. B. Wang, X. Zhang, X. Yuan, and J. Teng, "Optical Coupling of Surface Plasmons between Graphene Sheets," Appl. Phys. Lett., 100 [13] 131111 (2012).

89. B. Wang, X. Zhang, F. J. García-Vidal, X. Yuan, and J. Teng, "Strong Coupling of Surface Plasmon Polaritons in Monolayer Graphene Sheet Arrays," Phys. Rev. Lett., 109 [7] 073901 (2012).

90. A. Y. Nikitin, F. Guinea, F. J. García-Vidal, and L. Martín-Moreno, "Edge and Waveguide Terahertz Surface Plasmon Modes in Graphene Microribbons," Phys. Rev. B, 84 [16] 161407 (2011).

91. J. Christensen, A. Manjavacas, S. Thongrattanasiri, F. H. Koppens, and F. J. García de Abajo, "Graphene Plasmon Waveguiding and Hybridization in Individual and Paired Nanoribbons," ACS Nano., 6 [1] 431-40 (2011).

92. A. Y. Nikitin, F. Guinea, F. J. Garcia-Vidal, and L. Martin-Moreno, "Surface Plasmon Enhanced Absorption and Suppressed Transmission in Periodic Arrays of Graphene ribbons," Phys. Rev. B, 85 [8] 081405 (2012).

93. B. Sensale-Rodriguez, R. Yan, M. M. Kelly, T. Fang, K. Tahy, W. S. Hwang, D. Jena, L. Liu, and H. G. Xing, "Broadband Graphene Terahertz Modulators Enabled by Intraband Transitions," Nat. Commun., 3780 (2012).

94. S. Thongrattanasiri, F. H. Koppens, and F. J. G. De Abajo, "Complete Optical Absorption in Periodically Patterned Graphene," Phys. Rev. Lett., 108 [4] 047401 (2012).

95. Y. Fan, F. Zhang, Q. Zhao, Z. Wei, and H. Li, "Tunable Terahertz Coherent Perfect Absorption in a Monolayer Graphene," Opt. Lett., 39 [21] 6269-72 (2014).

96. Y. Fan, N.-H. Shen, T. Koschny, and C. M. Soukoulis, "Tunable Terahertz Meta-Surface with Graphene CutWires," ACS Photonics, 2 [1] 151-56 (2015).

97. Y. Fan, Z. Wei, Z. Zhang, and H. Li, "Enhancing Infrared Extinction and Absorption in a Monolayer Graphene Sheet by Harvesting the Electric Dipolar Mode of Split Ring Resonators," Opt. Lett., 38 [24] 5410-13 (2013).

98. T. V. Teperik, F. G. De Abajo, A. Borisov, M. Abdelsalam, P. Bartlett, Y. Sugawara, and J. Baumberg, "Omnidirectional Absorption in Nanostructured Metal Surfaces," Nature Photon., 2 [5] 299-301 (2008).

99. M. S. Jang, V. W. Brar, M. C. Sherrott, J. J. Lopez, L. Kim, S. Kim, M. Choi, and H. A. Atwater, "Tunable Large Resonant Absorption in a Midinfrared Graphene Salisbury Screen," Phys. Rev. B, 90 [16] 165409 (2014).

100. R. Alaee, M. Farhat, C. Rockstuhl, and F. Lederer, "A Perfect Absorber Made of a Graphene Micro-Ribbon Metamaterial," Opt. Express, 20 [27] 28017-24 (2012).
101. A. Andryieuski and A. V. Lavrinenko, "Graphene Metamaterials based Tunable Terahertz Absorber: Effective Surface Conductivity Approach," Opt. Express, 21 [7] 914455 (2013).

102. P.-Y. Chen, H. Huang, D. Akinwande, and A. Alú, "Graphene-based Plasmonic Platform for Reconfigurable Terahertz Nanodevices," ACS Photonics, 1 [8] 647-54 (2014).

103. S. F. Shi, B. Zeng, H. L. Han, X. Hong, H. Z. Tsai, H. S. Jung, A. Zettl, M. F. Crommie, and F. Wang, "Optimizing Broadband Terahertz Modulation with Hybrid Graphene/ Metasurface Structures," Nano Lett., 15 [1] 372-77 (2015).

104. Q. Li, L. Cong, R. Singh, N. Xu, W. Cao, X. Zhang, Z. Tian, L. Du, J. Han, and W. Zhang, "Monolayer Graphene Sensing Enabled by the Strong Fano-Resonant Metasurface," Nanoscale, 8 [39] 17278-84 (2016).

105. Q. Li, Z. Tian, X. Zhang, N. Xu, R. Singh, J. Gu, P. Lv, L.B. Luo, S. Zhang, J. Han, and W. Zhang, "Dual Control of Active Graphene-Silicon Hybrid Metamaterial Devices," Carbon, 90 146-53 (2015).

106. N. K. Emani, T.-F. Chung, X. Ni, A. V. Kildishev, Y. P. Chen, and A. Boltasseva, "Electrically Tunable Damping of Plasmonic Resonances with Graphene," Nano Lett., 12 [10] 5202-6 (2012).

107. Y. Yao, M. A. Kats, P. Genevet, N. Yu, Y. Song, J. Kong, and F. Capasso, "Broad Electrical Tuning of GrapheneLoaded Plasmonic Antennas," Nano Lett., 13 [3] 1257-64 (2013).

108. Y. Yao, R. Shankar, P. Rauter, Y. Song, J. Kong, M. Loncar, and F. Capasso, "High-Responsivity Mid-Infrared Graphene Detectors with Antenna-Enhanced Photocarrier Generation and Collection," Nano Lett., 14 [7] 3749-54 (2014).

109. S. H. Mousavi, I. Kholmanov, K. B. Alici, D. Purtseladze, N. Arju, K. Tatar, D. Y. Fozdar, J. W. Suk, Y. Hao, and A. B. Khanikaev, "Inductive Tuning of Fano-Resonant Metasurfaces Using Plasmonic Response of Graphene in the Mid-Infrared," Nano Lett., 13 [3] 1111-17 (2013).

110. D. A. Smirnova, A. E. Miroshnichenko, Y. S. Kivshar, and A. B. Khanikaev, "Tunable Nonlinear Graphene Metasurfaces," Phys. Rev. B, 92 [16] 161406 (2015).

111. Y. Yao, M. A. Kats, R. Shankar, Y. Song, J. Kong, M. Loncar, and F. Capasso, "Wide Wavelength Tuning of Optical Antennas on Graphene with Nanosecond Response Time," Nano Lett., 14 [1] 214-19 (2013).

112. B. Luk'yanchuk, N. I. Zheludev, S. A. Maier, N. J. Halas, P. Nordlander, H. Giessen, and C. T. Chong, "The Fano Resonance in Plasmonic Nanostructures and Metamaterials," Nature Mater., 9 [9] 707-15 (2010).

113. A. E. Miroshnichenko, S. Flach, and Y. S. Kivshar, "Fano Resonances in Nanoscale Structures," Rev. Mod. Phys., 82 [3] 2257 (2010).

114. B. Zhao, J. Zhao, and Z. Zhang, "Enhancement of NearInfrared Absorption in Graphene with Metal Gratings," Appl. Phys. Lett., 105 [3] 031905 (2014).

115. A. Boltasseva and H. A. Atwater, "Low-Loss Plasmonic Metamaterials," Science, 331 [6015] 290-91 (2011).

116. P. R. West, S. Ishii, G. V. Naik, N. K. Emani, V. M. Shalaev, and A. Boltasseva, "Searching for Better Plasmonic 
Materials," Laser Photonics Rev., 4 [6] 795-808 (2010).

117. C. Argyropoulos, "Enhanced Transmission Modulation Based on Dielectric Metasurfaces Loaded with Graphene," Opt. Express, 23 [18] 23787-97 (2015).

118. C. Wu, N. Arju, G. Kelp, J. A. Fan, J. Dominguez, E. Gonzales, E. Tutuc, I. Brener, and G. Shvets, "Spectrally Selective Chiral Silicon Metasurfaces based on Infrared Fano Resonances," Nat. Commun., 27 [5] 3892 (2014).

119. J. Zhang, W. Liu, Z. Zhu, X. Yuan, and S. Qin, "Strong Field Enhancement and Light-Matter Interactions with All-Dielectric Metamaterials based on Split Bar Resonators," Opt. Express, 22 [25] 30889-98 (2014).

120. S. Wu, S. Buckley, A. M. Jones, J. S. Ross, N. J. Ghimire, J. Yan, D. G. Mandrus, W. Yao, F. Hatami, and J. Vučković, "Control of Two-Dimensional Excitonic Light Emission via Photonic Crystal," 2D Mater., 1 [1] 011001 (2014).

121. R. Li, M. Imran, X. Lin, H. Wang, Z. Xu, and H. Chen, "Hybrid Airy Plasmons with Dynamically Steerable Trajectories," Nanoscale, 9 [4] 1449-56 (2017).

122. R.-J. Shiue, X. Gan, Y. Gao, L. Li, X. Yao, A. Szep, D. Walker Jr, J. Hone, and D. Englund, "Enhanced Photodetection in Graphene-Integrated Photonic Crystal Cavity," Appl. Phys. Lett., 103 [24] 241109 (2013).

123. A. Majumdar, J. Kim, J. Vuckovic, and F. Wang, "Electrical Control of Silicon Photonic Crystal Cavity by Graphene," Nano Lett., 13 [2] 515-18 (2013).

124. Y. Fan, Z. Wei, H. Li, H. Chen, and C. M. Soukoulis, "Pho- tonic Band Gap of a Graphene-Embedded Quarter-Wave Stack," Phys. Rev. B, 88 [24] 241403 (2013).

125. R. Li, H. Wang, B. Zheng, S. Dehdashti, E. Li, and H. Chen, "Bistable Scattering in Graphene-Coated Dielectric Nanowires," Nanoscale, 9 8449-57 (2017).

126. R. Li, X. Lin, S. Lin, X. Zhang, E. Li, and H. Chen, "Graphene Induced Mode Bifurcation at Low Input Power," Carbon, 98 463-67 (2016).

127. R. Li, X. Lin, S. Lin, X. Liu, and H. Chen, "Tunable DeepSubwavelength Superscattering Using Graphene Monolayers," Opt. Lett., 40 [8] 1651-54 (2015).

128. R. Li, X. Lin, S. Lin, X. Liu, and H. Chen, "Atomically Thin Spherical Shell-Shaped Superscatterers based on a Bohr Model," Nanotechnology, 26 [50] 505201 (2015).

129. R. Li, B. Zheng, X. Lin, R. Hao, S. Lin, W. Yin, E. Li, and H. Chen, "Design of Ultracompact Graphene-Based Superscatterers," IEEE J. Sel. Top. Quantum Electron., 23 [1] 130-37 (2017).

130. Y. Liu, R. Cheng, L. Liao, H. Zhou, J. Bai, G. Liu, L. Liu, Y. Huang, and X. Duan, "Plasmon Resonance Enhanced Multicolour Photodetection by Graphene," Nat. Commun., 2579 (2011).

131. T. J. Echtermeyer, L. Britnell, P. K. Jasnos, A. Lombardo, R. V. Gorbachev, A. N. Grigorenko, A. K. Geim, A. C. Ferrari, and K. S. Novoselov, "Strong Plasmonic Enhancement of Photovoltage in Graphene," Nat. Commun., 2458 (2011). 